\title{
Stochastic Control for Jump Diffusions *
}

\author{
Jingtao Shi
}

Additional information is available at the end of the chapter

http://dx.doi.org/10.5772/45719

\section{Introduction}

In this chapter, we will discuss the stochastic optimal control problem for jump diffusions. That is, the controlled stochastic system is driven by both Brownian motion and Poisson random measure and the controller wants to minimize/maximize some cost functional subject to the above stated state equation (stochastic control system) over the admissible control set. This kind of stochastic optimal control problems can be encountered naturally when some sudden and rare breaks take place, such as in the practical stock price market. An admissible control is called optimal if it achieves the infimum/supremum of the cost functional and the corresponding state variable and the cost functional are called the optimal trajectory and the value function, respectively.

It is well-known that Pontryagin's maximum principle (MP for short) and Bellman's dynamic programming principle (DPP for short) are the two principal and most commonly used approaches in solving stochastic optimal control problems. In the statement of maximum principle, the necessary condition of optimality is given. This condition is called the maximum condition which is always given by some Hamiltonian function. The Hamiltonian function is defined with respect to the system state variable and some adjoint variables. The equation that the adjoint variables satisfy is called adjoint equation, which is one or two backward stochastic differential equations (BSDEs for short) of [13]'s type. The system which consists of the adjoint equation, the original state equation, and the maximum condition is referred to as a generalized Hamiltonian system. On the other hand, the basic idea of dynamic programming principle is to consider a family of stochastic optimal control problems with different initial time and states and establish relationships among these problems via the so-called Hailton-Jacobi-Bellman (HJB for short) equation, which is a nonlinear second-order partial differential equation (PDE for short). If the HJB equation is solvable, we can obtain an optimal control by taking the maximizer/miminizer of the generalized Hamiltonian function involved in the HJB equation. To a great extent these two approaches have been developed separately and independently during the research in stochastic optimal control problems.

\footnotetext{
*The main content of this chapter is from the following published article paper: Shi, J.T., \& Wu, Z. (2011). Relationship between MP and DPP for the stochastic optimal control problem of jump diffusions. Applied mathematics and Optimization, Vol. 63, 151-189.
} 
Hence, a natural question arises: Are there any relations between these two methods? In fact, the relationship between MP and DPP is essentially the relationship between the adjoint processes and the value function, or the Hamiltonian systems and the HJB equations or even more generally, the relationship between stochastic differential equations (SDEs for short) and PDEs. Such a topic was intuitively discussed by [5], [4] and [9]. However, an important issue in studying the problem is that the derivatives of the value functions are unavoidably involved in these results. In fact, the value functions are usually not necessarily smooth. [19] first obtained the nonsmooth version of the relationship between MP and DPP using the viscosity solution and the second-order adjoint equation. See also the book by [18].

The aim of this chapter is to establish the relationship between MP and DPP within the framework of viscosity solutions in the jump diffusion setting. In this case, the state trajectory is described by a stochastic differential equation with Poisson jumps (SDEP for short). That is to say, the system noise (or the uncertainty of the problem) comes from a Brownian motion and a Poisson random measure. See [15] for theory and applications of this kind of equations. [16] proved the general MP where the control variable is allowed into both diffusion and jump coefficients. HJB equation for optimal control of jump diffusions can be seen in [12], which here is a second-order partial integral-differential equation (PIDE for short). [7] gave a sufficient MP by employing Arrow's generalization of the Mangasarian sufficient condition to the jump diffusion setting. Moreover, on the assumption that the value function is smooth, they showed the adjoint processes' connections to the value function. Let us state some results of [7] in detail with a slight modification to adapt to our setting.

A sufficient MP was proved to say that, for any admissible pair $\left(\bar{x}^{s, y ;} \bar{u}(\cdot), \bar{u}(\cdot)\right)$, if there exists an adapted solution $(p(\cdot), q(\cdot), \gamma(\cdot, \cdot))$ of the following adjoint equtaion

$$
\left\{\begin{aligned}
-d p(t)= & H_{x}\left(t, \bar{x}^{s, y ; \bar{u}}(t), \bar{u}(t), p(t), q(t), \gamma(t, \cdot)\right) d t-q(t) d W(t) \\
& -\int_{\mathbf{E}} \gamma(t, e) \tilde{N}(d e d t), t \in[0, T], \\
p(T)= & -h_{x}\left(\bar{x}^{s, y ; \bar{u}}(T)\right),
\end{aligned}\right.
$$

which is a BSDE with Poisson jumps (BSDEP for short) such that

$$
H\left(t, \bar{x}^{s, y ; \bar{u}}(t), \bar{u}(t), p(t), q(t), \gamma(t, \cdot)\right)=\sup _{u \in \mathbf{U}} H\left(t, \bar{x}^{s, y ; \bar{u}}(t), u, p(t), q(t), \gamma(t, \cdot)\right)
$$

for all $t \in[0, T]$ and that

$$
\hat{H}(x):=\max _{u \in \mathbf{U}} H(t, x, u, p(t), q(t), \gamma(t, \cdot))
$$

exists and is a concave function of $x$ for all $t \in[0, T]$, then $\left(\bar{x}^{s, y ;} \bar{u}(\cdot), \bar{u}(\cdot)\right)$ is an optimal pair. In the above, the Hamiltonian function $H:[0, T] \times \mathbf{R}^{n} \times \mathbf{U} \times \mathbf{R}^{n} \times \mathbf{R}^{n \times d} \times \mathcal{L}^{2}\left(\mathbf{E}, \mathcal{B}(\mathbf{E}), \pi ; \mathbf{R}^{n}\right) \rightarrow$ $\mathbf{R}$ is defined as

$$
H(t, x, u, p, q, \gamma(\cdot)):=f(t, x, u)+\langle p, b(t, x, u)\rangle+\operatorname{tr}\left\{\sigma(t, x, u)^{\top} q\right\}+\int_{\mathbf{E}}\langle\gamma(e), c(t, x, u, e)\rangle \pi(d e) .
$$

On the other hand, a DPP asserted that if the value function $V(\cdot, \cdot)$ belongs to $C^{1,2}\left([0, T] \times \mathbf{R}^{n}\right)$, then it satisfies the following HJB equation

$$
\sup _{u \in \mathbf{U}} F(t, x, u)=F(t, x, \bar{u}(t))=0
$$


where

$$
\begin{aligned}
F(t, x, u):= & f(t, x, u)+V_{t}(t, x)+\left\langle V_{x}(t, x), b(t, x, u)\right\rangle+\frac{1}{2} \operatorname{tr}\left\{V_{x x}(t, x) \sigma(t, x, u) \sigma(t, x, u)^{\top}\right\} \\
& +\int_{\mathbf{E}}\left[V(t, x+c(t, x, u, e))-V(t, x)-\left\langle V_{x}(t, x), c(t, x, u, e)\right\rangle\right] \pi(d e) .
\end{aligned}
$$

Moreover, Theorem 2.1 of [7] says that if $V(\cdot, \cdot)$ belongs to $C^{1,3}\left([0, T] \times \mathbf{R}^{n}\right)$, then the processes defined by

$$
\left\{\begin{aligned}
p(t) & =-V_{x}\left(t, \bar{x}^{s, y ; \bar{u}}(t)\right), \\
q(t) & =-V_{x x}\left(t, \bar{x}^{s, y ;} \bar{u}(t)\right) \sigma\left(t, \bar{x}^{s, y ; \bar{u}}(t), \bar{u}(t)\right), \\
\gamma(t, \cdot) & =-V_{x}\left(t, \bar{x}^{s, y ; \bar{u}}(t-)+c\left(t, \bar{x}^{s, y ; \bar{u}}(t-), \bar{u}(t)\right)+V_{x}\left(t, \bar{x}^{s, y ; \bar{u}}(t-)\right),\right.
\end{aligned}\right.
$$

solve the adjoint equation (1).

However, it seems that the above HJB equation (2) and the relationship (3) lack generality, since they require the value function to be smooth, which is not true even in the simplest case; see Example 3.2 of this chapter. This is an important gap in the literature [7]. The aim of this chapter is to bridge this gap by employing the notion of semijets evoked in defining the viscosity solutions.

The contribution of this chapter is as follows. Firstly, we give some basic properties of the value function and prove that the DPP still holds in our jump diffusion setting. Then we give the corresponding generalized HJB equation which now is a second-order PIDE. Secondly, we investigate the relationship between MP and DPP without assuming the continuous differentiablity of the value function. We obtain the relationship among the adjoint processes, the generalized Hamiltonian and the value function by employing the notions of the set-valued semijets evoked in defining the viscosity solutions, which is now interpreted as a set inclusion form among subjet, superjet of the value function, set contain adjoint processes and some "G-Gunction" (see the definition in Section 2). It is worth to pointed out that the controlled jump diffusions bring much technique difficulty to obtain the above results. In fact, the solution of the control system is not continuous with jump diffusions. We overcome these difficulty and get the desired results in this chapter which have wide applicable background.

The rest of this chapter is organized as follows. In Section 2, for stochastic optimal control problem of jump diffusions, we give some basic properties of the value function and then set out the corresponding DPP and MP, respectively. In Section 3, the relationship between MP and DPP is proved using the notion of viscosity solutions of PIDEs. Some concluding remarks are given in Section 4 .

\section{Problem statement and preliminaries}

Throughout this chapter, we denote by $\mathbf{R}^{n}$ the space of $n$-dimensional Euclidean space, by $\mathbf{R}^{n \times d}$ the space of matrices with order $n \times d$, by $\mathcal{S}^{n}$ the space of symmetric matrices with order $n \times n .\langle\cdot, \cdot\rangle$ and $|\cdot|$ denote the scalar product and norm in the Euclidean space, respectively. $T$ appearing in the superscripts denotes the transpose of a matrix. $a \vee b$ denotes $\max \{a, b\}$. C always denotes some positive constant. 
Let $\mathbf{E} \subset \mathbf{R}^{l}$ be a nonempty Borel set equipped with its Borel field $\mathcal{B}(\mathbf{E})$. Let $\pi(\cdot)$ be a bounded positive measure on $(\mathbf{E}, \mathcal{B}(\mathbf{E}))$. We denote by $\mathcal{L}^{2}\left(\mathbf{E}, \mathcal{B}(\mathbf{E}), \pi ; \mathbf{R}^{n}\right)$ or $\mathcal{L}^{2}$ the set of square integrable functions $k(\cdot): \mathbf{E} \rightarrow \mathbf{R}^{n}$ such that $\|\left. k(\cdot)\right|_{\mathcal{L}^{2}} ^{2}:=\int_{\mathbf{E}}|k(e)|^{2} \pi(d e)<\infty$.

Let $T>0$ and let $(\Omega, \mathcal{F}, \mathbf{P})$ be a complete probability space, equipped with a $d$-dimensional standard Brownian motion $\{W(t)\}_{0 \leq t \leq T}$ and a Poisson random measure $N(\cdot, \cdot)$ independent of $W(\cdot)$ with the intensity measure $\hat{N}(d e d t)=\pi(d e) d t$. We write $\tilde{N}(d e d t):=N(d e d t)-$ $\pi(d e) d t$ for the compensated Poisson martingale measure.

For a given $s \in[0, T)$, we suppose the filtration $\left\{\mathcal{F}_{t}^{s}\right\}_{s \leq t \leq T}$ is generated as the following

$$
\mathcal{F}_{t}^{s}:=\sigma\{N(\mathbf{A} \times(s, r]) ; s \leq r \leq t, \mathbf{A} \in \mathcal{B}(\mathbf{E})\} \bigvee \sigma\{W(r)-W(s) ; s \leq r \leq t\} \bigvee \mathcal{N},
$$

where $\mathcal{N}$ contains all P-null sets in $\mathcal{F}$ and $\sigma_{1} \bigvee \sigma_{2}$ denotes the $\sigma$-field generated by $\sigma_{1} \cup \sigma_{2}$. In particular, if $s=0$ we write $\mathcal{F}_{t} \equiv \mathcal{F}_{t}^{s}$.

Let $\mathbf{U}$ be a nonempty Borel subset of $\mathbf{R}^{k}$. For any initial time $s \in[0, T)$ and initial state $y \in \mathbf{R}^{n}$, we consider the following stochastic control system which is called a controlled jump diffusion process

$$
\left\{\begin{aligned}
d x^{s, y ; u}(t)= & b\left(t, x^{s, y ; u}(t), u(t)\right) d t+\sigma\left(t, x^{s, y ; u}(t), u(t)\right) d W(t) \\
& +\int_{\mathbf{E}} c\left(t, x^{s, y ; u}(t-), u(t), e\right) \tilde{N}(\text { dedt }), \quad t \in(s, T], \\
x^{s, y ; u}(s)= & y .
\end{aligned}\right.
$$

Here $b:[0, T] \times \mathbf{R}^{n} \times \mathbf{U} \rightarrow \mathbf{R}^{n}, \sigma:[0, T] \times \mathbf{R}^{n} \times \mathbf{U} \rightarrow \mathbf{R}^{n \times d}, c:[0, T] \times \mathbf{R}^{n} \times \mathbf{U} \times \mathbf{E} \rightarrow \mathbf{R}^{n}$ are given functions.

For a given $s \in[0, T)$, we denote by $\mathcal{U}[s, T]$ the set of $\mathbf{U}$-valued $\mathcal{F}_{t}^{s}$-predictable processes. For given $u(\cdot) \in \mathcal{U}[s, T]$ and $y \in \mathbf{R}^{n}$, a $\mathbf{R}^{n}$-valued process $x^{s, y ; u}(\cdot)$ is called a solution of (4) if it is an $\mathcal{F}_{t}^{s}$-adapted RCLL (i.e., right-continuous with left-hand limits) process such that (4) holds. It is called the state trajectory corresponding to the control $u(\cdot) \in \mathcal{U}[s, T]$ with initial state $y$. We refer to such $u(\cdot) \in \mathcal{U}[s, T]$ as an admissible control and $\left(x^{s, y ; u}(\cdot), u(\cdot)\right)$ as an admissible pair. For any $s \in[0, T)$, we introduce the following notations.

$$
\begin{aligned}
L^{2}\left(\Omega, \mathcal{F}_{T}^{s} ; \mathbf{R}^{n}\right):= & \left\{\mathbf{R}^{n} \text {-valued } \mathcal{F}_{T}^{s} \text {-measurable random variables } \xi ; \mathbb{E}|\xi|^{2}<\infty\right\}, \\
L_{\mathcal{F}}^{2}\left([s, T] ; \mathbf{R}^{n}\right):= & \left\{\mathbf{R}^{n} \text {-valued } \mathcal{F}_{t}^{s} \text {-adapted processes } \varphi(t) ; \mathbb{E} \int_{s}^{T}|\varphi(t)|^{2} d t<\infty\right\}, \\
L_{\mathcal{F}, p}^{2}\left([s, T] ; \mathbf{R}^{n}\right):= & \left\{\mathbf{R}^{n} \text {-valued } \mathcal{F}_{t}^{s} \text {-predictable processes } \phi(t) ; \mathbb{E} \int_{\mathcal{s}}^{T}|\phi(t)|^{2} d t<\infty\right\}, \\
F_{p}^{2}\left([s, T] ; \mathbf{R}^{n}\right):= & \left\{\mathbf{R}^{n} \text {-valued } \mathcal{F}_{t}^{s} \text {-predictable vector processes } \psi(t, e)\right. \text { defined on } \\
& \left.\Omega \times[0, T] \times \mathbf{E} ; \mathbb{E} \int_{s}^{T} \int_{\mathbf{E}}|\psi(t, e)|^{2} \pi(d e) d t<\infty\right\} .
\end{aligned}
$$

We consider the following cost functional

$$
J(s, y ; u(\cdot))=\mathbb{E}\left[\int_{s}^{T} f\left(t, x^{s, y ; u}(t), u(t)\right) d t+h\left(x^{s, y ; u}(T)\right)\right],
$$


where $f:[0, T] \times \mathbf{R}^{n} \times \mathbf{U} \rightarrow \mathbf{R}, h: \mathbf{R}^{n} \rightarrow \mathbf{R}$ are given functions. For given $(s, y) \in[0, T) \times \mathbf{R}^{n}$, the stochastic optimal control problem is to minimize (5) subject to (4) over $\mathcal{U}[s, T]$. An admissible pair $\left(\bar{x}^{s, y ; \bar{u}}(\cdot), \bar{u}(\cdot)\right)$ is called optimal if $\bar{u}(\cdot)$ achieves the infimum of $J(s, y ; u(\cdot))$ over $\mathcal{U}[s, T]$. We define the value function $V:[0, T] \times \mathbf{R}^{n} \rightarrow \mathbf{R}$ as

$$
\left\{\begin{aligned}
V(s, y) & =\inf _{u(\cdot) \in \mathcal{U}[s, T])} J(s, y ; u(\cdot)), \quad \forall(s, y) \in[0, T) \times \mathbf{R}^{n}, \\
V(T, y) & =h(y), \quad \forall y \in \mathbf{R}^{n} .
\end{aligned}\right.
$$

In this section, we first give some basic properties of the value function. Then we prove that the DPP still holds and introduce the generalized HJB equation and the generalized Hamiltonian function. The idea of proof is originated from [17], [18] while on some different assumptions. Then we introduce the Hamiltonian function, the $\mathcal{H}$-function and adjoint processes, then give the MP which is a special case of [16].

We first discuss the DPP and make the following assumptions.

(H1) $b, \sigma, c$ are uniformly continuous in $(t, x, u)$. There exists a constant $C>0$, such that

$$
\left\{\begin{array}{l}
|b(t, x, u)-b(t, \hat{x}, \hat{u})|+|\sigma(t, x, u)-\sigma(t, \hat{x}, \hat{u})| \\
\quad+|| c(t, x, u, \cdot)-c(t, \hat{x}, \hat{u}, \cdot)||_{\mathcal{L}^{2}} \leq C(|x-\hat{x}|+|u-\hat{u}|), \\
|b(t, x, u)|+|\sigma(t, x, u)|+|| c(t, x, u, \cdot)||_{\mathcal{L}^{2}} \leq C(1+|x|), \quad \forall t \in[0, T], x, \hat{x} \in \mathbf{R}^{n}, u, \hat{u} \in \mathbf{U} .
\end{array}\right.
$$

(H2) $f, h$ are uniformly continuous in $(t, x, u)$. There exists a constant $C>0$ and an increasing, continuous function $\bar{\omega}_{0}:[0, \infty) \times[0, \infty) \rightarrow[0, \infty)$ which satisfies $\bar{\omega}_{0}(r, 0)=0, \forall r \geq 0$, such that

$$
\left\{\begin{array}{l}
|f(t, x, u)-f(t, \hat{x}, u)|+|h(x)-h(\hat{x})| \leq \bar{\omega}_{0}(|x| \vee|\hat{x}|,|x-\hat{x}|), \\
|f(t, 0, u)|,|h(0)| \leq C, \quad \forall t \in[0, T], x, \hat{x} \in \mathbf{R}^{n}, u \in \mathbf{U}
\end{array}\right.
$$

Obviously, under assumption (H1), for any $u(\cdot) \in \mathcal{U}[s, T]$, SDEP (4) admits a unique solution $x^{s, y ; u}(\cdot)$ (see [10]).

Remark 2.1 We point out that assumption (H2) on $f, h$ allows them to have polynomial (in particular, quadratic) growth in $x$, provided that $b, \sigma, c$ have linear growth. A typical example is the stochastic linear quadratic (LQ for short) problem. Note that (H2) is different from assumptions (2.5), pp. 4 of [14] and assumption (S2)', pp. 248 of [18], where global Lipschitz condition is both imposed thus $f, h$ are global linear growth in $x$.

We need the following lemma.

Lemma 2.1 Let (H1) hold. For $k=2,4$, there exists $C>0$ such that for any $0 \leq s, \hat{s} \leq T, y, \hat{y} \in$ $\mathbf{R}^{n}, u(\cdot) \in \mathcal{U}[s, T]$,

$$
\begin{gathered}
\mathbb{E}\left|x^{s, y, u}(t)\right|^{k} \leq C\left(1+|y|^{k}\right), \quad t \in[s, T], \\
\mathbb{E}\left|x^{s, y, u}(t)-y\right|^{k} \leq C\left(1+|y|^{k}\right)(T-s)^{\frac{k}{2}}, \quad t \in[s, T], \\
\mathbb{E}\left[\sup _{s \leq t \leq T}\left|x^{s, y, u}(t)-y\right|\right]^{k} \leq C\left(1+|y|^{k}\right)(T-s)^{\frac{k}{2}}, \\
\mathbb{E}\left|x^{s, y, u}(t)-x^{s, \hat{y}, u}(t)\right|^{k} \leq C|y-\hat{y}|^{k}, \quad t \in[s, T], \\
\mathbb{E}\left|x^{s, y, u}(t)-x^{\hat{s}, y, u}(t)\right|^{k} \leq C(1+|y|)|s-\hat{s}|^{\frac{k}{2}}, \quad t \in[s \vee \hat{s}, T] .
\end{gathered}
$$


Estimates of the moments for SDEPs are proved in Lemma 3.1 of [14] for $k \in[0,2]$. In fact, under assumption (H1) we can easily extend his result to the case $k=2,4$ by virtue of Buckholder-Davis-Gundy's inquality. We leave the detail of the proof to the interested reader.

We give some basic continuity properties of the value function $V$. The proof is similar to Proposition 2.2, Chapter 2 of [17]. We omit the detail.

Proposition 2.1 Let $\mathbf{( H 1 )}, \mathbf{( H 2 )}$ hold. Then there exist increasing, continuous functions $\bar{\omega}_{1}$ : $[0, \infty) \rightarrow[0, \infty), \bar{\omega}_{2}:[0, \infty) \times[0, \infty) \rightarrow[0, \infty)$ which satisfies $\bar{\omega}_{2}(r, 0)=0, \forall r \geq 0$, such that

$$
\begin{gathered}
|V(s, y)| \leq \bar{\omega}_{1}(|y|), \quad \forall(s, y) \in[0, T) \times \mathbf{R}^{n}, \\
|V(s, y)-V(\hat{s}, \hat{y})| \leq \bar{\omega}_{2}\left(|y| \vee|\hat{y}|,|y-\hat{y}|+|\hat{s}-s|^{\frac{1}{2}} \mid\right), \quad \forall s, \hat{s} \in[0, T), y, \hat{y} \in \mathbf{R}^{n} .
\end{gathered}
$$

The following result is a version of Bellman's principle of optimality for jump diffusions.

Theorem 2.1 Suppose that (H1), (H2) hold. Then for any $(s, y) \in[0, T) \times \mathbf{R}^{n}$,

$$
V(s, y)=\inf _{u(\cdot) \in \mathcal{U}[s, T]} \mathbb{E}\left[\int_{s}^{\hat{s}} f\left(t, x^{s, y ; u}(t), u(t)\right) d t+V\left(\hat{s}, x^{s, y ; u}(\hat{s})\right)\right], \quad \forall 0 \leq s \leq \hat{s} \leq T .
$$

The proof is similar to Theorem 3.3, Chapter 4 of [18] or Proposition 3.2 of [14]. We omit it here.

The following result is to get the generalized HJB equation and its proof is similar to Proposition 3.4, Chapter 4 of [18].

Theorem 2.2 Suppose that (H1), (H2) hold and the value function $V \in C^{1,2}\left([0, T] \times \mathbf{R}^{n}\right)$. Then $V$ is a solution of the following generalized HJB equation which is a second-order PIDE:

$$
\left\{\begin{array}{l}
-V_{t}(t, x)+\sup _{u \in \mathbf{U}} G\left(t, x, u,-V(t, x),-V_{x}(t, x),-V_{x x}(t, x)\right)=0, \quad(t, x) \in[0, T) \times \mathbf{R}^{n}, \\
V(T, x)=h(x), \quad x \in \mathbf{R}^{n},
\end{array}\right.
$$

where, associated with a $\Psi \in C^{1,2}\left([0, T] \times \mathbf{R}^{n}\right)$, the generalized Hamiltonian function $G:[0, T] \times$ $\mathbf{R}^{n} \times \mathbf{U} \times \mathbf{R} \times \mathbf{R}^{n} \times \mathcal{S}^{n} \rightarrow \mathbf{R}$ is defined by

$$
\begin{aligned}
& G\left(t, x, u, \Psi(t, x), \Psi_{x}(t, x), \Psi_{x x}(t, x)\right) \\
& :=\left\langle\Psi_{x}(t, x), b(t, x, u)\right\rangle+\frac{1}{2} \operatorname{tr}\left\{\Psi_{x x}(t, x) \sigma(t, x, u) \sigma(t, x, u)^{\top}\right\} \\
& \quad-f(t, x, u)-\int_{\mathbf{E}}\left[\Psi(t, x+c(t, x, u, e))-\Psi(t, x)+\left\langle\Psi_{x}(t, x), c(t, x, u, e)\right\rangle\right] \pi(d e) .
\end{aligned}
$$

In the following, we discuss the stochastic MP and need the following hypothesis.

(H3) $b, \sigma, c, f, h$ are twice continuously differentiable in $x$, and $b_{x}, b_{x x}, \sigma_{x}, \sigma_{x x}, f_{x x}, h_{x x}$, $\left\|c_{x}(\cdot)\right\|_{\mathcal{L}^{2}},\left\|c_{x x}(\cdot)\right\|_{\mathcal{L}^{2}}$ are bounded. There exists a constant $C>0$ and a modulus of continuity $\bar{\omega}:[0, \infty) \rightarrow[0, \infty)$ such that 


$$
\left\{\begin{array}{l}
\left|b_{x}(t, x, u)-b_{x}(t, \hat{x}, \hat{u})\right|+\left|\sigma_{x}(t, x, u)-\sigma_{x}(t, \hat{x}, \hat{u})\right| \leq C|x-\hat{x}|+\bar{\omega}(|u-\hat{u}|), \\
|| c_{x}(t, x, u, \cdot)-c_{x}(t, \hat{x}, \hat{u}, \cdot)||_{\mathcal{L}^{2}} \leq C|x-\hat{x}|+\bar{\omega}(|u-\hat{u}|), \\
\left|b_{x x}(t, x, u)-b_{x x}(t, \hat{x}, \hat{u})\right|+\left|\sigma_{x x}(t, x, u)-\sigma_{x x}(t, \hat{x}, \hat{u})\right| \leq \bar{\omega}(|x-\hat{x}|+|u-\hat{u}|), \\
|| c_{x x}(t, x, u, \cdot)-c_{x x}(t, \hat{x}, \hat{u}, \cdot)||_{\mathcal{L}^{2}} \leq \bar{\omega}(|x-\hat{x}|+|u-\hat{u}|), \\
\left|f_{x}(t, x, u)\right|+\left|h_{x}(x)\right| \leq C(1+|x|), \\
|f(t, x, u)|+|h(x)| \leq C\left(1+|x|^{2}\right), \quad \forall t \in[0, T], x, \hat{x} \in \mathbf{R}^{n}, u, \hat{u} \in \mathbf{U} .
\end{array}\right.
$$

For simplicity, we introduce the following notations.

$$
\begin{aligned}
\bar{b}(t) & :=b\left(t, \bar{x}^{s, y ; \bar{u}}(t), \bar{u}(t)\right), \quad \bar{\sigma}(t):=\sigma\left(t, \bar{x}^{s, y ; j}(t), \bar{u}(t)\right), \\
\bar{f}(t) & :=f\left(t, \bar{x}^{s, y ; \bar{u}}(t), \bar{u}(t)\right), \quad \bar{c}(t, \cdot):=c\left(t, \bar{x}^{s, y ; \bar{u}}(t-), \bar{u}(t), \cdot\right), \\
\Delta \sigma(t ; u) & :=\sigma\left(t, \bar{x}^{s, y ; \bar{u}}(t), u\right)-\bar{\sigma}(t), \quad \Delta c(t, \cdot ; u):=c\left(t, \bar{x}^{s, y ; \bar{u}}(t-), u, \cdot\right)-\bar{c}(t, \cdot),
\end{aligned}
$$

and similar notations used for all their derivatives, for all $t \in[0, T], u \in \mathbf{U}$.

We define the Hamiltonian function $H:[0, T] \times \mathbf{R}^{n} \times \mathbf{U} \times \mathbf{R}^{n} \times \mathbf{R}^{n \times d} \times \mathcal{L}^{2}\left(\mathbf{E}, \mathcal{B}(\mathbf{E}), \pi ; \mathbf{R}^{n}\right) \rightarrow \mathbf{R}$ :

$$
\begin{aligned}
H(t, x, u, p, q, \gamma(\cdot)):= & \langle p, b(t, x, u)\rangle+\operatorname{tr}\left\{q^{\top} \sigma(t, x, u)\right\}-f(t, x, u) \\
& +\int_{\mathbf{E}}\langle\gamma(e), c(t, x, u, e)\rangle \pi(d e) .
\end{aligned}
$$

Associated with an optimal pair $\left(\bar{x}^{s, y ; \bar{u}}(\cdot), \bar{u}(\cdot)\right)$, we introduce the following first-order and second-order adjoint equations, respectively:

$$
\begin{gathered}
\left\{\begin{aligned}
-d p(t)=H_{x}\left(t, \bar{x}^{s, y ;} \bar{u}(t), \bar{u}(t), p(t), q(t), \gamma(t, \cdot)\right) d t-q(t) d W(t) \\
\quad-\int_{\mathbf{E}} \gamma(t, e) \tilde{N}(d e d t), t \in[s, T], \\
p(T)=-h_{x}\left(\bar{x}^{s, y ; \bar{u}}(T)\right),
\end{aligned}\right. \\
\left\{\begin{aligned}
-d P(t)= & \left\{\bar{b}_{x}(t)^{\top} P(t)+P(t) \bar{b}_{x}(t)+\bar{\sigma}_{x}(t)^{\top} P(t) \bar{\sigma}_{x}(t)+\bar{\sigma}_{x}(t)^{\top} Q(t)+Q(t) \bar{\sigma}_{x}(t)\right. \\
& +\int_{\mathbf{E}}\left[\bar{c}_{x}(t, e)^{\top} P(t) \bar{c}_{x}(t, e)+\bar{c}_{x}(t, e)^{\top} R(t, e) \bar{c}_{x}(t, e)+\bar{c}_{x}(t, e)^{\top} R(t, e)\right. \\
& \left.\left.+R(t, e) \bar{c}_{x}(t, e)\right] \pi(d e)+H_{x x}\left(t, \bar{x}^{s, y ;} \bar{u}(t), \bar{u}(t), p(t), q(t), \gamma(t, \cdot)\right)\right\} d t \\
- & Q(t) d W(t)-\int_{\mathbf{E}} R(t, e) \tilde{N}(d e d t), \quad t \in[s, T],
\end{aligned}\right. \\
P(T)=-h_{x x}\left(\bar{x}^{s, y ;}, \bar{u}(T)\right),
\end{gathered}
$$

Under (H1) (H3), by Lemma 2.4 of [16], we know that BSDEPs (18) and (19) admit unique adapted solutions $(p(\cdot), q(\cdot), \gamma(\cdot, \cdot))$ and $(P(\cdot), Q(\cdot), R(\cdot, \cdot))$ satisfying

$$
\left\{\begin{aligned}
(p(\cdot), q(\cdot), \gamma(\cdot, \cdot)) & \in L_{\mathcal{F}}^{2}\left([s, T] ; \mathbf{R}^{n}\right) \times L_{\mathcal{F}, p}^{2}\left([s, T] ; \mathbf{R}^{n \times d}\right) \times F_{p}^{2}\left([s, T] ; \mathbf{R}^{n}\right), \\
(P(\cdot), Q(\cdot), R(\cdot, \cdot)) & \in L_{\mathcal{F}}^{2}\left([s, T] ; \mathcal{S}^{n}\right) \times\left(L_{\mathcal{F}, p}^{2}\left([s, T] ; \mathcal{S}^{n}\right)\right)^{d} \times F_{p}^{2}\left([s, T] ; \mathcal{S}^{n}\right) .
\end{aligned}\right.
$$


Note that $p(\cdot)$ and $P(\cdot)$ are RCLL processes. Associated with an optimal pair $\left(\bar{x}^{s, y ;} \bar{u}(\cdot), \bar{u}(\cdot)\right)$ and its corresponding adjoint processes $(p(\cdot), q(\cdot), \gamma(\cdot, \cdot))$ and $(P(\cdot), Q(\cdot), R(\cdot, \cdot))$ satisfying (20), we define an $\mathcal{H}$-function $\mathcal{H}:[0, T] \times \mathbf{R}^{n} \times \mathbf{U} \rightarrow \mathbf{R}$ as

$$
\begin{aligned}
\mathcal{H}(t, x, u):= & H(t, x, u, p(t), q(t), \gamma(t, \cdot))-\frac{1}{2} \operatorname{tr}\left\{P(t)\left[\bar{\sigma}(t) \bar{\sigma}(t)^{\top}+\int_{\mathbf{E}} \bar{c}(t, e) \bar{c}(t, e)^{\top} \pi(d e)\right]\right\} \\
& +\frac{1}{2} \operatorname{tr}\left\{P(t)\left[\Delta \sigma(t ; u) \Delta \sigma(t ; u)^{\top}+\int_{\mathbf{E}} \Delta c(t, e ; u) \Delta c(t, e ; u)^{\top} \pi(d e)\right]\right\} \\
& -\frac{1}{2} \operatorname{tr}\left\{\int_{\mathbf{E}} R(t, e)\left[\bar{c}(t, e) \bar{c}(t, e)^{\top}-\Delta c(t, e ; u) \Delta c(t, e ; u)^{\top}\right] \pi(d e)\right\} \\
\equiv & \langle p(t), b(t, x, u)\rangle+\operatorname{tr}\{q(t), \sigma(t, x, u)\}+\int_{\mathbf{E}}\langle\gamma(t, e), c(t, x, u, e)\rangle \pi(d e) \\
& -f(t, x, u)-\frac{1}{2} \operatorname{tr}\left\{P(t)\left[\bar{\sigma}(t) \bar{\sigma}(t)^{\top}+\int_{\mathbf{E}} \bar{c}(t, e) \bar{c}(t, e)^{\top} \pi(d e)\right]\right\} \\
& +\frac{1}{2} \operatorname{tr}\left\{P(t)\left[\Delta \sigma(t ; u) \Delta \sigma(t ; u)^{\top}+\int_{\mathbf{E}} \Delta c(t, e ; u) \Delta c(t, e ; u)^{\top} \pi(d e)\right]\right\} \\
& -\frac{1}{2} \operatorname{tr}\left\{\int_{\mathbf{E}} R(t, e)\left[\bar{c}(t, e) \bar{c}(t, e)^{\top}-\Delta c(t, e ; u) \Delta c(t, e ; u)^{\top}\right] \pi(d e)\right\} .
\end{aligned}
$$

The following result is the general stochastic MP for jump diffusions.

Theorem 2.3 Suppose that $\mathbf{( H 1 )} \sim(\mathbf{H} 3)$ hold. Let $(s, y) \in[0, T) \times \mathbf{R}^{n}$ be fixed and $\left(\bar{x}^{s, y ;} \bar{u}(\cdot), \bar{u}(\cdot)\right)$ be an optimal pair of our stochastic optimal control problem. Then there exist triples of processes $(p(\cdot), q(\cdot), \gamma(\cdot, \cdot))$ and $(P(\cdot), Q(\cdot), R(\cdot, \cdot))$ satisfying $(20)$ and they are solutions of first-order and second-order adjoint equations (18) and (19), respectively, such that the following maximum condition holds:

$$
\begin{aligned}
& H\left(t, \bar{x}^{s, y ; \bar{u}}(t), \bar{u}(t), p(t), q(t), \gamma(t, \cdot)\right)-H\left(t, \bar{x}^{s, y ; \bar{u}}(t), u, p(t), q(t), \gamma(t, \cdot)\right) \\
& -\frac{1}{2} \operatorname{tr}\left\{P(t)\left[\Delta \sigma(t ; u) \Delta \sigma(t ; u)^{\top}+\int_{\mathbf{E}} \Delta c(t, e ; u) \Delta c(t, e ; u)^{\top} \pi(d e)\right]\right\} \\
& -\frac{1}{2} \operatorname{tr}\left\{\int_{\mathbf{E}} R(t, e)\left[\Delta c(t, e ; u) \Delta c(t, e ; u)^{\top}\right] \pi(d e)\right\} \geq 0, \quad \forall u \in \mathbf{U}, \text { a.e.t } \in[s, T], \mathbf{P}-a . s .,
\end{aligned}
$$

or equivalently,

$$
\mathcal{H}(t, \bar{x}(t), \bar{u}(t))=\max _{u \in \mathbf{U}} \mathcal{H}(t, \bar{x}(t), u), \quad \text { a.e.t } \in[s, T], \quad \mathbf{P} \text {-a.s. }
$$

Proof It is an immediate consequence of Theorem 2.1 of [16]. The equivalence of (22) and (23) is obvious.

Remark 2.2 Note that the integrand with respect to the compensated martingale measure $\tilde{N}$ in the second-order adjoint equation enters into the above maximum condition, while the counterpart in the diffusion case does not! This marks one essential difference of the maximum principle between an optimally controlled diffusion (continuous) process and an optimally controlled jump (discontinuous) process. 


\section{Relationship between Stochastic MP and DPP}

In this section, we will establish the relationship between stochastic MP and DPP in the language of viscosity solutions. That is to say, we will consider the viscosity solutions of the generalized HJB equation (15). In our jump diffusion setting, we need use the viscosity solution theory for second-order PIDEs. For convenience, we refer to [1], [2], [14], [3], [11], [6] for a deep investigation of PIDEs in the framework of viscosity solutions. In Subsection 3.1, we first present some preliminary results concerning viscosity solutions and semijets. Then we give the relationship between stochastic MP and DPP in Subsection 3.2. Special cases on the assumption that the value function is smooth are given as corollaries. Some examples are also given to illustrate our results.

\subsection{Preliminary results: viscosity solutions and semijets}

To make the chapter self-contained, we present the definition of viscosity solutions and semijets, which is frequently seen in the literature and we state it adapting to the generalized HJB equation (15) in our jump diffusion setting.

Definition 3.1 (i) A function $v \in C\left([0, T] \times \mathbf{R}^{n}\right)$ is called a viscosity subsolution of (15) if $v(T, x) \leq h(x), \forall x \in \mathbf{R}^{n}$, and for any test function $\psi \in C^{1,2}\left([0, T] \times \mathbf{R}^{n}\right)$, whenever $v-\psi$ attains $a$ global maximum at $(t, x) \in[0, T) \times \mathbf{R}^{n}$, then

$$
-\psi_{t}(t, x)+\sup _{u \in \mathbf{U}} G\left(t, x, u,-\psi(t, x),-\psi_{x}(t, x),-\psi_{x x}(t, x)\right) \leq 0 .
$$

(ii) A function $v \in C\left([0, T] \times \mathbf{R}^{n}\right)$ is called a viscosity supersolution of (15) if $v(T, x) \geq h(x), \forall x \in$ $\mathbf{R}^{n}$, and for any test function $\psi \in C^{1,2}\left([0, T] \times \mathbf{R}^{n}\right)$, whenever $v-\psi$ attains a global minimum at $(t, x) \in[0, T] \times \mathbf{R}^{n}$, then

$$
-\psi_{t}(t, x)+\sup _{u \in \mathbf{U}} G\left(t, x, u,-\psi(t, x),-\psi_{x}(t, x),-\psi_{x x}(t, x)\right) \geq 0 .
$$

(iii) If $v \in C\left([0, T] \times \mathbf{R}^{n}\right)$ is both a viscosity subsolution and viscosity supersolution of (15), then it is called a viscosity solution of (15).

In order to give the existence and uniqueness result for viscosity solution of the generalized HJB equation (15), it is convenient to give an intrinsic characterization of viscosity solutions. Let us recall the right parabolic super-subjets of a continuous function on $[0, T] \times \mathbf{R}^{n}$ (see [18] or [3]). For $v \in C\left([0, T] \times \mathbf{R}^{n}\right)$ and $(\hat{t}, \hat{x}) \in[0, T) \times \mathbf{R}^{n}$, the right parabolic superjet of $v$ at $(\hat{t}, \hat{x})$ is the set triple

$$
\begin{aligned}
\mathcal{P}_{t+, x}^{1,2,+} v(\hat{t}, \hat{x}):=\{ & (q, p, P) \in \mathbf{R} \times \mathbf{R}^{n} \times \mathcal{S}^{n} \mid v(t, x) \leq v(\hat{t}, \hat{x})+q(t-\hat{t})+\langle p, x-\hat{x}\rangle \\
& \left.+\frac{1}{2}(x-\hat{x})^{\top} P(x-\hat{x})+o\left(|t-\hat{t}|+|x-\hat{x}|^{2}\right), \text { as } t \downarrow \hat{t}, x \rightarrow \hat{x}\right\},
\end{aligned}
$$

and the right parabolic subjet of $v$ at $(\hat{t}, \hat{x})$ is the set

$$
\begin{aligned}
\mathcal{P}_{t+, x}^{1,2,-} v(\hat{t}, \hat{x}):= & \left\{(q, p, P) \in \mathbf{R} \times \mathbf{R}^{n} \times \mathcal{S}^{n} \mid v(t, x) \geq v(\hat{t}, \hat{x})+q(t-\hat{t})+\langle p, x-\hat{x}\rangle\right. \\
& \left.+\frac{1}{2}(x-\hat{x})^{\top} P(x-\hat{x})+o\left(|t-\hat{t}|+|x-\hat{x}|^{2}\right), \text { as } t \downarrow \hat{t}, x \rightarrow \hat{x}\right\} .
\end{aligned}
$$


From the above definitions, we see immediately that

$$
\left\{\begin{array}{l}
\mathcal{P}_{t+, x}^{1,2,+} v(\hat{t}, \hat{x})+[0, \infty) \times\{0\} \times \mathcal{S}_{+}^{n}=\mathcal{P}_{t+, x}^{1,2,+} v(\hat{t}, \hat{x}), \\
\mathcal{P}_{t+, x}^{1,2,-} v(\hat{t}, \hat{x})-[0, \infty) \times\{0\} \times \mathcal{S}_{+}^{n}=\mathcal{P}_{t+, x}^{1,2,-} v(\hat{t}, \hat{x}),
\end{array}\right.
$$

where $\mathcal{S}_{+}^{n}:=\left\{S \in \mathcal{S}^{n} \mid S \geq 0\right\}$, and $A \pm B:=\{a \pm b \mid a \in A, b \in B\}$ for any subsets $A$ and $B$ in a same Euclidean space.

Remark 3.1 Suppose that $\psi \in C^{1,2}\left([0, T] \times \mathbf{R}^{n}\right)$. If $v-\psi$ attains a global maximum at $(\hat{t}, \hat{x}) \in$ $[0, T) \times \mathbf{R}^{n}$, then

$$
\left(\psi_{t}(\hat{t}, \hat{x}), \psi_{x}(\hat{t}, \hat{x}), \psi_{x x}(\hat{t}, \hat{x})\right) \in \mathcal{P}_{t+, x}^{1,2,+} v(\hat{t}, \hat{x}) .
$$

If $v-\psi$ attains a global minimum at $(\hat{t}, \hat{x}) \in[0, T) \times \mathbf{R}^{n}$, then

$$
\left(\psi_{t}(\hat{t}, \hat{x}), \psi_{x}(\hat{t}, \hat{x}), \psi_{x x}(\hat{t}, \hat{x})\right) \in \mathcal{P}_{t+, x}^{1,2,-} v(\hat{t}, \hat{x}) .
$$

The following result is useful and whose proof for diffusion case can be found, for instance, in Lemma 5.4, Chapter 4 of [18].

Proposition 3.1 Let $v \in C\left([0, T] \times \mathbf{R}^{n}\right)$ and $\left(t_{0}, x_{0}\right) \in[0, T) \times \mathbf{R}^{n}$ be given. Then

(i) $(q, p, P) \in \mathcal{P}_{t+, x}^{1,2,+} v\left(t_{0}, x_{0}\right)$ if and only if there exist a function $\psi \in C^{1,2}\left([0, T] \times \mathbf{R}^{n}\right)$, such that

$$
\left\{\begin{array}{l}
\left(\psi\left(t_{0}, x_{0}\right), \psi_{t}\left(t_{0}, x_{0}\right), \psi_{x}\left(t_{0}, x_{0}\right), \psi_{x x}\left(t_{0}, x_{0}\right)\right)=\left(v\left(t_{0}, x_{0}\right), q, p, P\right), \\
\psi(t, x)>v(t, x), \quad \forall\left(t_{0}, x_{0}\right) \neq(t, x) \in\left[t_{0}, T\right] \times \mathbf{R}^{n} .
\end{array}\right.
$$

(ii) $(q, p, P) \in \mathcal{P}_{t+, x}^{1,2,-} v\left(t_{0}, x_{0}\right)$ if and only if there exist a function $\psi \in C^{1,2}\left([0, T] \times \mathbf{R}^{n}\right)$, such that

$$
\left\{\begin{array}{l}
\left(\psi\left(t_{0}, x_{0}\right), \psi_{t}\left(t_{0}, x_{0}\right), \psi_{x}\left(t_{0}, x_{0}\right), \psi_{x x}\left(t_{0}, x_{0}\right)\right)=\left(v\left(t_{0}, x_{0}\right), q, p, P\right), \\
\psi(t, x)<v(t, x), \quad \forall\left(t_{0}, x_{0}\right) \neq(t, x) \in\left[t_{0}, T\right] \times \mathbf{R}^{n} .
\end{array}\right.
$$

We will also make use of the partial super-subjets with respect to one of the variables $t$ and $x$. Therefore, we need the following definitions.

$$
\left\{\begin{aligned}
\mathcal{P}_{x}^{2,+} v(\hat{t}, \hat{x}):=\{ & (p, P) \in \times \mathbf{R}^{n} \times \mathcal{S}^{n} \mid v(\hat{t}, x) \leq v(\hat{t}, \hat{x})+\langle p, x-\hat{x}\rangle \\
& \left.+\frac{1}{2}(x-\hat{x})^{\top} P(x-\hat{x})+o\left(|x-\hat{x}|^{2}\right), \text { as } x \rightarrow \hat{x}\right\}, \\
\mathcal{P}_{x}^{2,-} v(\hat{t}, \hat{x}):=\{ & (p, P) \in \times \mathbf{R}^{n} \times \mathcal{S}^{n} \mid v(\hat{t}, x) \geq v(\hat{t}, \hat{x})+\langle p, x-\hat{x}\rangle \\
& \left.+\frac{1}{2}(x-\hat{x})^{\top} P(x-\hat{x})+o\left(|x-\hat{x}|^{2}\right), \text { as } x \rightarrow \hat{x}\right\},
\end{aligned}\right.
$$

and

$$
\left\{\begin{array}{l}
\mathcal{P}_{t+}^{1,+} v(\hat{t}, \hat{x}):=\{q \in \mathbf{R} \mid v(t, \hat{x}) \leq v(\hat{t}, \hat{x})+q(t-\hat{t})+o(|t-\hat{t}|), \text { as } t \downarrow \hat{t}\}, \\
\mathcal{P}_{t+}^{1,-} v(\hat{t}, \hat{x}):=\{q \in \mathbf{R} \mid v(t, \hat{x}) \geq v(\hat{t}, \hat{x})+q(t-\hat{t})+o(|t-\hat{t}|), \text { as } t \downarrow \hat{t}\} .
\end{array}\right.
$$


Using the above definitions, we can give the following intrinsic formulation of viscosity solution of the generalized HJB equation (15).

Definition 3.2 (i) A function $v \in C\left([0, T] \times \mathbf{R}^{n}\right)$ satisfying $v(T, x) \leq h(x), \forall x \in \mathbf{R}^{n}$ is a viscosity subsolution of (15) if, for any test function $\psi \in C^{1,2}\left([0, T] \times \mathbf{R}^{n}\right)$, if $v-\psi$ attains a global maximum at $(t, x) \in[0, T) \times \mathbf{R}^{n}$ and if $(q, p, P) \in \mathcal{P}_{t+, x}^{1,2,+} v(t, x)$ with $q=\psi_{t}(t, x), p=\psi_{x}(t, x), P \leq \psi_{x x}(t, x)$, then

$$
-q+\sup _{u \in \mathbf{U}}\{G(t, x, u,-\psi(t, x),-p,-P)\} \leq 0 .
$$

(ii) A function $v \in C\left([0, T] \times \mathbf{R}^{n}\right)$ satisfying $v(T, x) \geq h(x), \forall x \in \mathbf{R}^{n}$ is a viscosity supersolution of (15) if, for any test function $\psi \in C^{1,2}\left([0, T] \times \mathbf{R}^{n}\right)$, if $v-\psi$ attains a global minimum at $(t, x) \in$ $[0, T) \times \mathbf{R}^{n}$ and if $(q, p, P) \in \mathcal{P}_{t+, x}^{1,2,-} v(t, x)$ with $q=\psi_{t}(t, x), p=\psi_{x}(t, x), P \geq \psi_{x x}(t, x)$, then

$$
-q+\sup _{u \in \mathbf{U}}\{G(t, x, u,-\psi(t, x),-p,-P)\} \geq 0 .
$$

(iii) If $v \in C\left([0, T] \times \mathbf{R}^{n}\right)$ is both a viscosity subsolution and viscosity supersolution of (15), then it is called a viscosity solution of (15).

Proposition 3.2 Definitions 3.1 and 3.2 are equivalent.

Proof The result is immediate in view of Proposition 3.1. In fact it is a special case of Proposition 1 of [3].

The following result is the existence and uniqueness of viscosity solution of the generalized HJB equation (15).

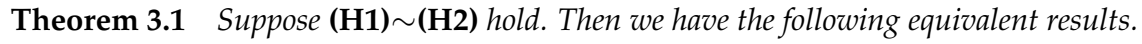

(i) The value function $V \in C\left([0, T] \times \mathbf{R}^{n}\right)$ defined by (6) is a unique viscosity solution of (15) in the class of functions satisfying (12), (13).

(ii) The value function $V \in C\left([0, T] \times \mathbf{R}^{n}\right)$ is the only function that satisfies (12), (13) and the following: For all $(t, x) \in[0, T) \times \mathbf{R}^{n}$,

$$
\left\{\begin{array}{l}
-q+\sup _{u \in \mathbf{U}}\left\{G\left(t, x, u,-\psi_{1}(t, x),-p,-P\right)\right\} \leq 0, \quad \forall(q, p, P) \in \mathcal{P}_{t+, x}^{1,2,+} V(t, x), \\
\psi_{1} \in C^{1,2}\left([0, T] \times \mathbf{R}^{n}\right) \text { such that } \psi_{1}\left(t^{\prime}, x^{\prime}\right)>v\left(t^{\prime}, x^{\prime}\right), \forall\left(t^{\prime}, x^{\prime}\right) \neq(t, x) \in[t, T] \times \mathbf{R}^{n}, \\
-q+\sup _{u \in \mathbf{U}}\left\{G\left(t, x, u,-\psi_{2}(t, x),-p,-P\right)\right\} \geq 0, \quad \forall(q, p, P) \in \mathcal{P}_{t+, x}^{1,2,-} V(t, x), \\
\psi_{2} \in C^{1,2}\left([0, T] \times \mathbf{R}^{n}\right) \text { such that } \psi_{2}\left(t^{\prime}, x^{\prime}\right)<v\left(t^{\prime}, x^{\prime}\right), \forall\left(t^{\prime}, x^{\prime}\right) \neq(t, x) \in[t, T] \times \mathbf{R}^{n}, \\
V(T, x)=h(x) .
\end{array}\right.
$$

Proof Result ( $i$ ) is a special case of Theorems 3.1 and 4.1 of [14]. Result (ii) is obvious by virtue of Propositions 3.1 and 3.2. The equivalence between ( $i)$ and $(i i)$ is obvious. 


\subsection{Main results: Relationship between Stochastic MP and DPP}

Proposition 2.1 tell us that the value function has nice continuity properties. But in general we cannot obtain the differentiablity of it. Therefore we should not suppose the generalized HJB equation (15) always admits an enough smooth (classic) solution. In fact it is not true even in the simplest case; see Example 3.2 in this subsection. This is an important gap in the literature (see Section 2, [7], for example). Fortunately, this gap can be bridged by the theory of viscosity solutions. This is one of the main contributions of this paper.

The following result shows that the adjoint process $p, P$ and the value function $V$ relate to each other within the framework of the superjet and the subjet in the state variable $x$ along an optimal trajectory.

Theorem 3.2 Suppose $\mathbf{( H 1 )} \sim(\mathbf{H} 3)$ hold and let $(s, y) \in[0, T) \times \mathbf{R}^{n}$ be fixed. Let $\left(\bar{x}^{s, y ;} \bar{u}(\cdot), \bar{u}(\cdot)\right)$ be an optimal pair of our stochastic optimal control problem. Let $(p(\cdot), q(\cdot), \gamma(\cdot, \cdot))$ and $(P(\cdot), Q(\cdot), R(\cdot, \cdot))$ are first-order and second-order adjoint processes, respectively. Then

$$
\begin{array}{ll}
\{-p(t)\} \times[-P(t), \infty) \subseteq \mathcal{P}_{x}^{2,+} V\left(t, \bar{x}^{s, y ; \bar{u}}(t)\right), \quad \forall t \in[s, T], \quad \mathbf{P} \text {-a.s., } \\
\mathcal{P}_{x}^{2,-} V\left(t, \bar{x}^{s, y ; \bar{u}}(t)\right) \subseteq\{-p(t)\} \times(-\infty, P(t)], \quad \forall t \in[s, T], \quad \mathbf{P} \text {-a.s. }
\end{array}
$$

We also have

$$
\mathcal{P}_{x}^{1,-} V\left(t, \bar{x}^{s, y ; \bar{u}}(t)\right) \subseteq\{-p(t)\} \subseteq \mathcal{P}_{x}^{1,+} V\left(t, \bar{x}^{s, y ; \bar{u}}(t)\right), \quad \forall t \in[s, T], \quad \text { P-a.s. }
$$

Proof Fix a $t \in[s, T]$. For any $z \in \mathbf{R}^{n}$, denote by $x^{t, z ; \bar{u}}(\cdot)$ the solution of the following SDEP:

$$
\begin{aligned}
x^{t, z ; \bar{u}}(r)= & z+\int_{t}^{r} b\left(r, x^{t, z ; \bar{u}}(r), \bar{u}(r)\right) d r+\int_{t}^{r} \sigma\left(r, x^{t, z ; \bar{u}}(r), \bar{u}(r)\right) d W(r) \\
& +\int_{\mathbf{E}} \int_{t}^{r} c\left(r, x^{t, z ; \bar{u}}(r-), \bar{u}(r), e\right) \tilde{N}(d e d r), \quad r \in[t, T] .
\end{aligned}
$$

It is clear that (37) can be regarded as an SDEP on $\left(\Omega, \mathcal{F},\left\{\mathcal{F}_{r}^{s}\right\}_{r \geq s}, \mathbf{P}\left(\cdot \mid \mathcal{F}_{t}^{s}\right)(\omega)\right)$ for P-a.s. $\omega$, where $\mathbf{P}\left(\cdot \mid \mathcal{F}_{t}^{s}\right)(\omega)$ is the regular conditional probability given $\mathcal{F}_{t}^{s}$ defined on $(\Omega, \mathcal{F})$ (see pp. 12-16 of [10]). In probability space $\left(\Omega, \mathcal{F}, \mathbf{P}\left(\cdot \mid \mathcal{F}_{t}^{s}\right)(\omega)\right)$, random variable $\bar{x}^{s, y ; \bar{u}}(t, \omega)$ is almost surely a constant vector in $\mathbf{R}^{n}$ (we still denote it by $\bar{x}^{s, y ;} \bar{u}(t, \omega)$ ).

Set $\xi^{t, z ; \bar{u}}(r):=x^{t, z ; \bar{u}}(r)-\bar{x}^{t, \bar{x}^{s, y ; \bar{u}}(t) ; \bar{u}}(r), t \leq r \leq T$. Thus by Lemma 2.1 we have

$$
\mathbb{E}\left[\sup _{t \leq r \leq T}\left|\xi^{t, z ; \bar{u}}(r)\right|^{k} \mid \mathcal{F}_{t}^{s}\right](\omega) \leq C\left|z-\bar{x}^{s, y ; \bar{u}}(t, \omega)\right|^{k}, \quad \text { P-a.s.w., } \quad \text { for } k=2,4 .
$$

Now we rewrite the equation for $\xi^{t, z ; \bar{u}}(\cdot)$ in two different ways based on different orders of expansion, which called the first-order and second-order variational equations, respectively:

$$
\left\{\begin{aligned}
d \xi^{t, z ; \bar{u}}(r)= & \bar{b}_{x}(r) \xi^{t, z ; \bar{u}}(r) d r+\bar{\sigma}_{x}(r) \xi^{t, z ; \bar{u}}(r) d W(r)+\int_{\mathbf{E}} \bar{c}_{x}(r, e) \xi^{t, z ; \bar{u}}(r-) \tilde{N}(\text { ded } r) \\
& +\varepsilon_{z 1}(r) d r+\varepsilon_{z 2}(r) d W(r)+\int_{\mathbf{E}} \varepsilon_{z 3}(r, e) \tilde{N}(\text { ded } r), \quad r \in[t, T], \\
\xi^{t, z ; \bar{u}}(t)= & z-\bar{x}^{s, y ; \bar{u}}(t),
\end{aligned}\right.
$$


where

$$
\left\{\begin{aligned}
\varepsilon_{z 1}(r) & :=\int_{0}^{1}\left[b_{x}\left(r, \bar{x}^{s, y ; \bar{u}}(r)+\theta \xi^{t, z ; \bar{u}}(r), \bar{u}(r)\right)-\bar{b}_{x}(r)\right] \xi^{t, z ; \bar{u}}(r) d \theta, \\
\varepsilon_{z 2}(r) & :=\int_{0}^{1}\left[\sigma_{x}\left(r, \bar{x}^{s, y ; \bar{u}}(r)+\theta \xi^{t, z ; \bar{u}}(r), \bar{u}(r)\right)-\bar{\sigma}_{x}(r)\right] \xi^{t, z ; \bar{u}}(r) d \theta, \\
\varepsilon_{z 3}(r, \cdot) & :=\int_{0}^{1}\left[c_{x}\left(r, \bar{x}^{s, y ; \bar{u}}(r-)+\theta \xi^{t, z ; \bar{u}}(r-), \bar{u}(r), \cdot\right)-\bar{c}_{x}(r, \cdot)\right] \xi^{t, z ; \bar{u}}(r-) d \theta,
\end{aligned}\right.
$$

and

$$
\left\{\begin{aligned}
d \xi^{t, z ; \bar{u}}(r)= & \left\{\bar{b}_{x}(r) \xi^{t, z ; \bar{u}}(r)+\frac{1}{2} \xi^{t, z ; \bar{u}}(r)^{\top} \bar{b}_{x x}(r) \xi^{t, z ; \bar{u}}(r)\right\} d r \\
+ & \left\{\bar{\sigma}_{x}(r) \xi^{t, z ; \bar{u}}(r)+\frac{1}{2} \xi^{t, z ; \bar{u}}(r)^{\top} \bar{\sigma}_{x x}(r) \xi^{t, z ; \bar{u}}(r)\right\} d W(r) \\
+ & \int_{\mathbf{E}}\left\{\bar{c}_{x}(r, e) \xi^{t, z ; \bar{u}}(r-)+\frac{1}{2} \xi^{t, z ; \bar{u}}(r-)^{\top} \bar{c}_{x x}(r, e) \xi^{t, z ; \bar{u}}(r-)\right\} \tilde{N}(d e d r) \\
& +\varepsilon_{z 4}(r) d r+\varepsilon_{z 5}(r) d W(r)+\int_{\mathbf{E}} \varepsilon_{z 6}(r, e) \tilde{N}(d e d r), \quad r \in[t, T], \\
\xi^{t, z ; \bar{u}}(t)= & z-x^{s, y ; \bar{u}}(t),
\end{aligned}\right.
$$

where

$$
\left\{\begin{aligned}
\varepsilon_{z 4}(r) & :=\int_{0}^{1}(1-\theta) \xi^{t, z ; \bar{u}}(r)^{\top}\left[b_{x x}\left(r, \bar{x}^{s, y ; \bar{u}}(r)+\theta \xi^{t, z ; \bar{u}}(r), \bar{u}(r)\right)-\bar{b}_{x x}(r)\right] \xi^{t, z ; \bar{u}}(r) d \theta, \\
\varepsilon_{z 5}(r) & :=\int_{0}^{1}(1-\theta) \xi^{t, z ; \bar{u}}(r)^{\top}\left[\sigma_{x x}\left(r, \bar{x}^{s, y ; \bar{u}}(r)+\theta \xi^{t, z ; \bar{u}}(r), \bar{u}(r)\right)-\bar{\sigma}_{x x}(r)\right] \xi^{t, z ; \bar{u}}(r) d \theta, \\
\varepsilon_{z 6}(r, \cdot) & :=\int_{0}^{1}(1-\theta) \xi^{t, z ; \bar{u}}(r-)^{\top}\left[c_{x x}\left(r, \bar{x}^{s, y ; \bar{u}}(r-)+\theta \xi^{t, z ; \bar{u}}(r-), \bar{u}(r), \cdot\right)-\bar{c}_{x x}(r, \cdot)\right] \xi^{t, z ; \bar{u}}(r-) d \theta .
\end{aligned}\right.
$$

We are going to show that, there exists a deterministic continuous and increasing function $\delta:[0, \infty) \rightarrow[0, \infty)$, independent of $z \in \mathbf{R}^{n}$, with $\frac{\delta(r)}{r} \rightarrow 0$ as $r \rightarrow 0$, such that

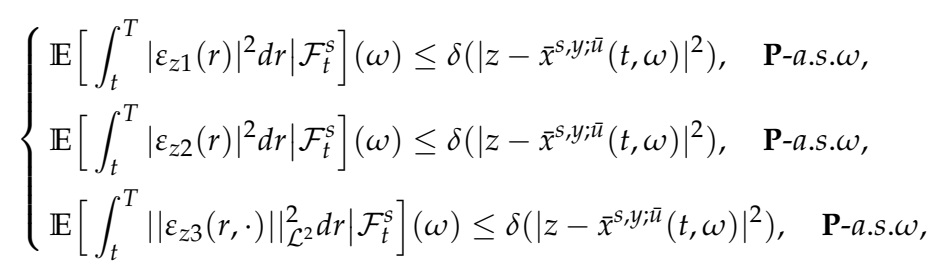

and

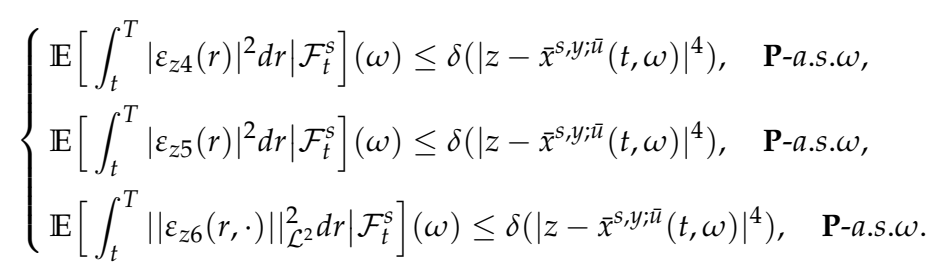


We start to prove (41). To this end, let us fixed an $\omega \in \Omega$ such that (38) holds. Then, by setting $b_{x}(r, \theta):=b_{x}\left(r, \bar{x}^{s, y ; \bar{u}}(r)+\theta \xi^{t, z ; \bar{u}}(r), \bar{u}(r)\right)$ and in virtue of (H3), we have

$$
\begin{aligned}
& \mathbb{E}\left[\int_{t}^{T}\left|\varepsilon_{z 1}(r)\right|^{2} d r \mid \mathcal{F}_{t}^{s}\right](\omega) \\
& \leq \int_{t}^{T} \mathbb{E}\left\{\int_{0}^{1}\left|b_{x}(r, \theta)-\bar{b}_{x}(r)\right|^{2} d \theta \cdot\left|\xi^{t, z ; \bar{u}}(r)\right|^{2} \mid \mathcal{F}_{t}^{s}\right\}(\omega) d r \\
& \leq C \int_{t}^{T} \mathbb{E}\left[\left|\xi^{t, z ; \bar{u}}(r)\right|^{4} \mid \mathcal{F}_{t}^{s}\right](\omega) d r \leq C\left|z-\bar{x}^{s, y ; \bar{u}}(t, \omega)\right|^{4} .
\end{aligned}
$$

Thus, the first inequality in (41) follows if we choose $\delta(r) \equiv C r^{2}, r \geq 0$. The second inequality in (41) can be proved similarly. Setting $c_{x}(r, \theta, \cdot):=c_{x}\left(r, \bar{x}^{s, y ;} \bar{u}(r-)+\theta \xi^{t, z ; \bar{u}}(r-), \bar{u}(r), \cdot\right)$ and using (H3), we have

$$
\begin{aligned}
& \mathbb{E}\left[\int_{t}^{T}\left\|\varepsilon_{z 3}(r, \cdot)\right\|_{\mathcal{L}^{2}}^{2} d r \mid \mathcal{F}_{t}^{s}\right](\omega) \\
& \leq \int_{t}^{T} \mathbb{E}\left\{\int_{0}^{1}|| c_{x}(r, \theta, \cdot)-\bar{c}_{x}(r, \cdot) \|_{\mathcal{L}^{2}}^{2} d \theta \cdot\left|\xi^{t, z ; \bar{u}}(r-)\right|^{2} \mid \mathcal{F}_{t}^{s}\right\}(\omega) d r \\
& \leq C \int_{t}^{T} \mathbb{E}\left[\left|\xi^{t, z ; \bar{u}}(r-)\right|^{4} \mid \mathcal{F}_{t}^{s}\right](\omega) d r \\
& =C \int_{t}^{T} \mathbb{E}\left[\left|\xi^{t, z ; \bar{u}}(r)\right|^{4} \mid \mathcal{F}_{t}^{s}\right](\omega) d r \leq C\left|z-\bar{x}^{s, y ; \bar{u}}(t, \omega)\right|^{4} .
\end{aligned}
$$

The equality in (43) holds because the discontinuous points of $\xi^{t, z ; \bar{u}}(\cdot)$ are at most countable. Thus, the third inequality in (41) follows for an obvious $\delta(\cdot)$ as above.

We continue to prove (42). Let $b_{x x}(r, \theta):=b_{x x}\left(r, \bar{x}^{s, y ; j \bar{u}}(r)+\theta \xi^{t, z ; \bar{u}}(r), \bar{u}(r)\right)$. Using (H3), we can show that

$$
\begin{aligned}
& \mathbb{E}\left[\int_{t}^{T}\left|\varepsilon_{z 4}(r)\right|^{2} d r \mid \mathcal{F}_{t}^{s}\right](\omega) \\
& \leq \int_{t}^{T} \mathbb{E}\left[\int_{0}^{1}\left|b_{x x}(r, \theta)-\bar{b}_{x x}(r)\right|^{2} d \theta \cdot\left|\xi^{t, z ; \bar{u}}(r)\right|^{4} \mid \mathcal{F}_{t}^{s}\right](\omega) d r \\
& \leq \int_{t}^{T}\left\{\mathbb{E}\left[\bar{\omega}\left(\left|\xi^{t, z ; \bar{u}}(r)\right|^{4}\right) \mid \mathcal{F}_{t}^{s}\right](\omega)\right\}^{\frac{1}{2}}\left\{\mathbb{E}\left[\left|\xi^{t, z ; \bar{u}}(r)\right|^{8} \mid \mathcal{F}_{t}^{s}\right](\omega)\right\}^{\frac{1}{2}} d r \\
& \leq C \int_{t}^{T}\left\{\mathbb{E}\left[\bar{\omega}\left(\left|\xi^{t, z ; \bar{u}}(r)\right|^{4}\right) \mid \mathcal{F}_{t}^{s}\right](\omega)\right\}^{\frac{1}{2}} d r \cdot\left|z-\bar{x}^{s, y ; \bar{u}}(t, \omega)\right|^{4} .
\end{aligned}
$$

This yields the first inequality in (42) if we choose $\delta(r) \equiv C r \sqrt{\bar{\omega}(r)}, r \geq 0$. Noting that the modulus of continuity $\bar{\omega}(\cdot)$ is defined in (H3). The second inequality in (42) can be proved similarly. Setting $c_{x x}(r, \theta, \cdot):=c_{x x}\left(r, \bar{x}^{s, y ; \bar{u}}(r-)+\theta \xi^{t, z ; \bar{u}}(r-), \bar{u}(r), \cdot\right)$ and by virtue of (H3), noting the remark following (43), we show that 


$$
\begin{aligned}
& \mathbb{E}\left[\int_{t}^{T}\left\|\varepsilon_{z 6}(r, \cdot)\right\|_{\mathcal{L}^{2}}^{2} d r \mid \mathcal{F}_{t}^{s}\right](\omega) \\
& \leq \int_{t}^{T} \mathbb{E}\left[\int_{0}^{1}|| c_{x x}(r, \theta, \cdot)-\bar{c}_{x x}(r, \cdot)||_{\mathcal{L}^{2}}^{2} d \theta \cdot\left|\xi^{t, z ; \bar{u}}(r-)\right|^{4} \mid \mathcal{F}_{t}^{s}\right](\omega) d r \\
& \leq \int_{t}^{T}\left\{\mathbb{E}\left[\bar{\omega}\left(\left|\xi^{t, z ; \bar{u}}(r-)\right|^{4}\right) \mid \mathcal{F}_{t}^{s}\right](\omega)\right\}^{\frac{1}{2}}\left\{\mathbb{E}\left[\left|\xi^{t, z ; \bar{u}}(r-)\right|^{8} \mid \mathcal{F}_{t}^{s}\right](\omega)\right\}^{\frac{1}{2}} d r \\
& \leq C \int_{t}^{T}\left\{\mathbb{E}\left[\bar{\omega}\left(\left|\xi^{t, z ; \bar{u}}(r)\right|^{4}\right) \mid \mathcal{F}_{t}^{s}\right](\omega)\right\}^{\frac{1}{2}} d r \cdot\left|z-\bar{x}^{s, y ; \bar{u}}(t, \omega)\right|^{4} .
\end{aligned}
$$

This yields the third inequality in (42) for an obvious $\delta(\cdot)$ as above. Finally, we can select the largest $\delta(\cdot)$ obtained in the above six calculations. For example, we can choose an enough large constant $C>0$ and define $\delta(r) \equiv \operatorname{Cr}(r \vee \sqrt{\bar{\omega}(r)}), r \geq 0$. Then (41), (42) follows with a $\delta(\cdot)$ independent of $z \in \mathbf{R}^{n}$.

Applying Itô's formula to $\left\langle\xi^{t, z ; \bar{u}}(\cdot), p(\cdot)\right\rangle$, noting (18) and (40), we have

$$
\begin{aligned}
& \mathbb{E}\left\{\int_{t}^{T}\left\langle\bar{f}_{x}(r), \xi^{t, z ; \bar{u}}(r)\right\rangle d r+\left\langle h_{x}\left(\bar{x}^{s, y ; \bar{u}}(T)\right), \xi^{t, z ; \bar{u}}(T)\right\rangle \mid \mathcal{F}_{t}^{s}\right\} \\
= & \left\langle-p(t), \xi^{t, z ; \bar{u}}(t)\right\rangle-\mathbb{E}\left\{\frac { 1 } { 2 } \int _ { t } ^ { T } \left[\left\langle p(r), \xi^{t, z ; \bar{u}}(r)^{\top} \bar{b}_{x x}(r) \xi^{t, z ; \bar{u}}(r)\right\rangle\right.\right. \\
& \left.+\left\langle q(r), \xi^{t, z ; \bar{u}}(r)^{\top} \bar{\sigma}_{x x}(r) \xi^{t, z ; \bar{u}}(r)\right\rangle+\int_{\mathbf{E}}\left\langle\gamma(r, e), \xi^{t, z ; \bar{u}}(r)^{\top} \bar{c}_{x x}(r, e) \xi^{t, z ; \bar{u}}(r)\right\rangle \pi(d e)\right] d r \\
& \left.-\int_{t}^{T}\left[\left\langle p(r), \varepsilon_{z 4}(r)\right\rangle+\left\langle q(r), \varepsilon_{z 5}(r)\right\rangle+\int_{\mathbf{E}}\left\langle\gamma(r, e), \varepsilon_{z 6}(r, e)\right\rangle \pi(d e)\right] d r \mid \mathcal{F}_{t}^{s}\right\}, \quad \mathbf{P}-a . s .
\end{aligned}
$$

On the other hand, apply Itô's formula to $\Phi^{t, z ; \bar{u}}(r):=\xi^{t, z ; \bar{u}}(r) \xi^{t, z ; \bar{u}}(r)^{\top}$, noting (39), we get

$$
\left\{\begin{aligned}
& d \Phi^{t, z ; \bar{u}}(r)=\left\{\bar{b}_{x}(r) \Phi^{t, z ; \bar{u}}(r)+\Phi^{t, z ; \bar{u}}(r) \bar{b}_{x}(r)^{\top}+\bar{\sigma}_{x}(r) \Phi^{t, z ; \bar{u}}(r) \bar{\sigma}_{x}(r)^{\top}\right. \\
&\left.+\int_{\mathbf{E}} \bar{c}_{x}(r, e) \Phi^{t, z ; \bar{u}}(r) \bar{c}_{x}(r, e)^{\top} \pi(d e)+\varepsilon_{z 7}(r)\right\} d r \\
&+\left\{\bar{\sigma}_{x}(r) \Phi^{t, z ; \bar{u}}(r)+\Phi^{z}(r) \bar{\sigma}_{x}(r)^{\top}+\varepsilon_{z 8}(r)\right\} d W(r) \\
&+\int_{\mathbf{E}}\left\{\bar{c}_{x}(r, e) \Phi^{t, z ; \bar{u}}(r-) \bar{c}_{x}(r, e)^{\top}+\bar{c}_{x}(r, e) \Phi^{t, z ; \bar{u}}(r-)\right. \\
&\left.+\Phi^{t, z ; \bar{u}}(r-) \bar{c}_{x}(r, e)^{\top}+\varepsilon_{z 9}(r, e)\right\} \tilde{N}(d e d r), \quad r \in[t, T], \\
& \Phi^{t, z ; \bar{u}}(t)=\xi^{t, z ; \bar{u}}(t) \xi^{t, z ; \bar{u}}(t)^{\top},
\end{aligned}\right.
$$


where

$$
\left\{\begin{aligned}
\varepsilon_{z 7}(r):= & \varepsilon_{z 1}(r) \xi^{t, z ; \bar{u}}(r)^{\top}+\xi^{t, z ; \bar{u}}(r) \varepsilon_{z 1}(r)^{\top}+\bar{\sigma}_{x}(r) \xi^{t, z ; \bar{u}}(r) \varepsilon_{z 2}(r)^{\top} \\
+ & \varepsilon_{z 2}(r) \xi^{t, z ; \bar{u}}(r)^{\top} \bar{\sigma}_{x}(r)^{\top}+\varepsilon_{z 2}(r) \varepsilon_{z 2}(r)^{\top}+\int_{\mathbf{E}}\left\{\bar{c}_{x}(r, e) \xi^{t, z ; \bar{u}}(r) \varepsilon_{z 3}(r, e)^{\top}\right. \\
& \left.+\varepsilon_{z 3}(r, e) \xi^{t, z ; \bar{u}}(r)^{\top} \bar{c}_{x}(r, e)^{\top}+\varepsilon_{z 3}(r, e) \varepsilon_{z 3}(r, e)^{\top}\right\} \pi(d e), \\
\varepsilon_{z 8}(r):= & \varepsilon_{z 2}(r) \xi^{t, z ; \bar{u}}(r)^{\top}+\xi^{t, z ; \bar{u}}(r) \varepsilon_{z 2}(r)^{\top}, \\
\varepsilon_{z 9}(r, \cdot):= & \bar{c}_{x}(r, \cdot) \xi^{t, z ; \bar{u}}(r-) \varepsilon_{z 3}(r, \cdot)^{\top}+\varepsilon_{z 3}(r, \cdot) \xi^{t, z ; \bar{u}}(r-)^{\top} \bar{c}_{x}(r, \cdot)^{\top} \\
& +\xi^{t, z ; \bar{u}}(r-) \varepsilon_{z 3}(r, \cdot)^{\top}+\varepsilon_{z 3}(r, \cdot) \xi^{t, z ; \bar{u}}(r-)^{\top}+\varepsilon_{z 3}(r, \cdot) \varepsilon_{z 3}(r, \cdot)^{\top} .
\end{aligned}\right.
$$

Once more applying Itô's formula to $\Phi^{t, z ; \bar{u}}(\cdot)^{\top} P(\cdot)$, noting (19) and (46), we get

$$
\begin{aligned}
& -\mathbb{E}\left\{\int_{t}^{T} \xi^{t, z ; \bar{u}}(r)^{\top} \bar{H}_{x x}(r) \xi^{t, z ; \bar{u}}(r) d r+\xi^{t, z ; \bar{u}}(T)^{\top} h_{x x}\left(\bar{x}^{s, y ; \bar{u}}(T)\right) \xi^{t, z ; \bar{u}}(T) \mid \mathcal{F}_{t}^{s}\right\} \\
= & -\xi^{t, z ; \bar{u}}(t)^{\top} P(t) \xi^{t, z ; \bar{u}}(t) \\
& -\mathbb{E}\left\{\int_{t}^{T} \operatorname{tr}\left\{P(r) \varepsilon_{z 7}(r)+Q(r) \varepsilon_{z 8}(r)+\int_{\mathbf{E}} R(r, e) \varepsilon_{z 9}(r, e) \pi(d e)\right\} d r \mid \mathcal{F}_{t}^{s}\right\}, \quad \text { P-a.s. }
\end{aligned}
$$

Let us call a $z \in \mathbf{R}^{n}$ rational if all its coordinate are rational. Since the set of all rational $z \in \mathbf{R}^{n}$ is countable, we may find a common subset $\Omega_{0} \subseteq \Omega$ with $P\left(\Omega_{0}\right)=1$ such that for any $\omega_{0} \in \Omega_{0}$,

$$
\left\{\begin{array}{l}
V\left(t, \bar{x}^{s, y ; \bar{u}}\left(t, \omega_{0}\right)\right)=\mathbb{E}\left[\int_{s}^{T} \bar{f}(r) d r+h\left(\bar{x}^{s, y ; \bar{u}}(T)\right) \mid \mathcal{F}_{t}^{s}\right]\left(\omega_{0}\right), \\
(38),(41),(42),(45),(47) \text { are satisfied for any rational } z \text {, and }\left.\bar{u}(\cdot)\right|_{[t, T]} \in \mathcal{U}[t, T] .
\end{array}\right.
$$

Let $\omega_{0} \in \Omega_{0}$ be fixed, then for any rational $z \in \mathbf{R}^{n}$, noting (41) and (42), we have

$$
\begin{aligned}
& V(t, z)-V\left(t, \bar{x}^{s, y ; \bar{u}}\left(t, \omega_{0}\right)\right) \\
= & \mathbb{E}\left\{\int_{t}^{T}\left[f\left(r, x^{t, z ; \bar{u}}(r), \bar{u}(r)\right)-\bar{f}(r)\right] d r+h\left(x^{t, z ; \bar{u}}(T)\right)-h\left(\bar{x}^{s, y ; \bar{u}}(T)\right) \mid \mathcal{F}_{t}^{s}\right\}\left(\omega_{0}\right) \\
= & \mathbb{E}\left\{\int_{t}^{T}\left\langle\bar{f}_{x}(r), \xi^{t, z ; \bar{u}}(r)\right\rangle+\left\langle h_{x}\left(\bar{x}^{s, y ;} ; \bar{u}(T)\right), \xi^{t, z ; \bar{u}}(T)\right\rangle \mid \mathcal{F}_{t}^{s}\right\}\left(\omega_{0}\right) \\
& +\frac{1}{2} \mathbb{E}\left\{\int_{t}^{T} \xi^{t, z ; \bar{u}}(r)^{\top} \bar{f}_{x x}(r) \xi^{t, z ; \bar{u}}(r) d r+\xi^{t, z ; \bar{u}}(T)^{\top} h_{x x}\left(\bar{x}^{s, y ; \bar{u}}(T)\right) \xi^{t, z ; \bar{u}}(T) \mid \mathcal{F}_{t}^{s}\right\}\left(\omega_{0}\right) \\
& +o\left(\left|z-\bar{x}^{s, y ; \bar{u}}\left(t, \omega_{0}\right)\right|^{2}\right) .
\end{aligned}
$$


By virtue of (45) and (47), we have

$$
\begin{aligned}
& V(t, z)-V\left(t, \bar{x}^{s, y ; \bar{u}}\left(t, \omega_{0}\right)\right) \\
\leq & -\left\langle p\left(t, \omega_{0}\right), \xi^{t, z ; \bar{u}}\left(t, \omega_{0}\right)\right\rangle-\frac{1}{2} \mathbb{E}\left\{\int_{t}^{T} \xi^{t, z ; \bar{u}}(r)^{\top} \bar{H}_{x x}(r) \xi^{t, z ; \bar{u}}(r) d r\right. \\
& \left.+\xi^{t, z ; \bar{u}}(T)^{\top} h_{x x}\left(\bar{x}^{s, y ; \bar{u}}(T)\right) \xi^{t, z ; \bar{u}}(T) \mid \mathcal{F}_{t}^{s}\right\}\left(\omega_{0}\right)+o\left(\left|z-\bar{x}^{s, y ; i \bar{u}}\left(t, \omega_{0}\right)\right|^{2}\right) \\
= & -\left\langle p\left(t, \omega_{0}\right), \xi^{t, z ; \bar{u}}\left(t, \omega_{0}\right)\right\rangle-\frac{1}{2} \xi^{t, z ; \bar{u}}\left(t, \omega_{0}\right)^{\top} P\left(t, \omega_{0}\right) \xi^{t, z ; \bar{u}}\left(t, \omega_{0}\right)+o\left(\left|z-\bar{x}^{s, y ; \bar{u}}\left(t, \omega_{0}\right)\right|^{2}\right) \\
= & -\left\langle p\left(t, \omega_{0}\right), z-\bar{x}^{s, y ; \bar{u}}\left(t, \omega_{0}\right)\right\rangle-\frac{1}{2}\left(z-\bar{x}^{s, y ; \bar{u}}\left(t, \omega_{0}\right)\right)^{\top} P\left(t, \omega_{0}\right)\left(z-\bar{x}^{s, y ; \bar{u}}\left(t, \omega_{0}\right)\right) \\
& +o\left(\left|z-\bar{x}^{s, y ; \bar{u}}\left(t, \omega_{0}\right)\right|^{2}\right) .
\end{aligned}
$$

Note that the term $o\left(\left|z-\bar{x}^{s, y ; \bar{u}}\left(t, \omega_{0}\right)\right|^{2}\right)$ depends only on the size of $\left|z-\bar{x}^{s, y ; \bar{u}}\left(t, \omega_{0}\right)\right|^{2}$, and it is independent of $z$. Therefore, by the continuity of $V(t, \cdot)$, we see that (49) holds for all $z \in \mathbf{R}^{n}$, which by definition (30) proves

$$
(-p(t),-P(t)) \in \mathcal{P}_{x}^{2,+} V\left(t, \bar{x}^{s, y ; \bar{u}}(t)\right), \quad \forall t \in[s, T], \quad \mathbf{P} \text {-a.s. }
$$

Then (34) holds. Let us now show (35). Fix an $\omega \in \Omega$ such that (49) holds for any $z \in \mathbf{R}^{n}$. For any $(p, P) \in \mathcal{P}_{x}^{2,-} V\left(t, \bar{x}^{s, y ;} \bar{u}(t)\right)$, by definition (30) and (49) we have

$$
\begin{aligned}
0 \leq & V(t, z)-V(t, \bar{x}(t, \omega))-\left\langle p, z-\bar{x}^{s, y ; \bar{u}}(t, \omega)\right\rangle-\frac{1}{2}\left(z-\bar{x}^{s, y ; \bar{u}}(t, \omega)\right)^{\top} P\left(z-\bar{x}^{s, y ; \bar{u}}(t, \omega)\right) \\
= & -\left\langle p(t, \omega)+p, z-\bar{x}^{s, y ; \bar{u}}(t, \omega)\right\rangle-\frac{1}{2}\left(z-\bar{x}^{s, y ; \bar{u}}(t, \omega)\right)^{\top}(P(t, \omega)+P)\left(z-\bar{x}^{s, y ; \bar{u}}(t, \omega)\right) \\
& +o\left(\left|z-\bar{x}^{s, y ; \bar{u}}(t, \omega)\right|^{2}\right) .
\end{aligned}
$$

Then, it is necessary that

$$
p=-p(t), \quad P \leq-P(t), \quad \mathbf{P} \text {-a.s. }
$$

Thus, (35) holds. (36) is immediate. The proof is complete.

Remark 3.2 It is interesting to note that if $V \in C^{1,2}\left([0, T] \times \mathbf{R}^{n}\right)$, then (34) (35) reduce to

$$
\left\{\begin{array}{l}
V_{x}\left(t, \bar{x}^{s, y ; \bar{u}}(t)\right)=-p(t), \\
V_{x x}\left(t, \bar{x}^{s, y ; \bar{u}}(t)\right) \leq-P(t), \quad \forall t \in[s, T], \quad \text { P-a.s. }
\end{array}\right.
$$

We point out that in our jump diffusion setting the strict inequality $V_{x x}\left(t, \bar{x}^{s, y ; \bar{u}}(t)\right)<-P(t)$ may happen in some cases, as shown in the following example.

Example 3.1 Consider the following linear stochastic control system with Poisson jumps $(n=1)$ :

$$
\left\{\begin{aligned}
d x^{s, y ; u}(t) & =u(t) d t+\delta u(t) \tilde{N}(d t), \quad t \in[s, T], \\
x^{s, y ; u}(s) & =y,
\end{aligned}\right.
$$

for some $\delta \neq 0$. Here $N$ is a Poisson process with the intensity $\lambda d t$ and $\tilde{N}(d t):=N(d t)-$ $\lambda d t(\lambda>0)$ is the compensated martingale measure. The quadratic cost functional is taken as

$$
J(s, y ; u(\cdot))=\frac{1}{2} \mathbb{E} \int_{s}^{T}\left(\frac{1}{\lambda}-\frac{1-\lambda}{\lambda} e^{\frac{t-T}{\delta^{2}}}\right) x^{s, y ; u}(t)^{2} d t .
$$


Define

$$
\phi(t)=\delta^{2}\left(1-e^{\frac{t-T}{\delta^{2}}}\right), \quad \forall t \in[0, T] .
$$

For any $u(\cdot) \in \mathcal{U}[s, T]$, applying Itô's formula to $\phi(t) x^{s, y ; u}(t)^{2}$, we have

$$
\begin{aligned}
d \phi(t) x^{s, y ; u}(t)^{2}= & \phi(t)\left(2 x^{s, y ; u}(t) u(t)+\lambda \delta^{2} u(t)^{2}\right) d t+x^{s, y ; u}(t)^{2}\left(\frac{\phi(t)}{\delta^{2}}-1\right) d t \\
& +\phi(t)\left(2 \delta x^{s, y ; u}(t-) u(t)+\delta^{2} u(t)^{2}\right) \tilde{N}(d t) .
\end{aligned}
$$

Integrating from $s$ to $T$, taking expectation on both sides, we have

$$
\begin{aligned}
0 & =\mathbb{E}\left[\phi(T) x^{s, y ; u}(T)^{2}\right] \\
& =\phi(s) y^{2}+\mathbb{E} \int_{s}^{T}\left[2 \phi(t) x^{s, y ; u}(t) u(t)+\lambda \delta^{2} \phi(t) u(t)^{2}+x^{s, y ; u}(t)^{2}\left(\frac{1}{\delta^{2}} \phi(t)-1\right)\right] d t .
\end{aligned}
$$

Thus

$$
J(s, y ; u(\cdot))=\frac{1}{2} \phi(s) y^{2}+\mathbb{E} \int_{s}^{T} \lambda \delta^{2} \phi(t)\left[u(t)+\frac{x^{s, y ; u}(t)}{\lambda \delta^{2}}\right]^{2} d t .
$$

This implies that

$$
\bar{u}(t)=-\frac{x^{s, y ; u}(t)}{\lambda \delta^{2}}, \quad \forall t \in[s, T],
$$

is a state feedback optimal control and the value function is

$$
V(s, y)=\frac{1}{2} \delta^{2}\left(1-e^{\frac{s-T}{\delta^{2}}}\right) y^{2}, \quad \forall(s, y) \in[0, T] \times \mathbf{R} .
$$

On the other hand, the second-order adjoint equation is

$$
\left\{\begin{array}{l}
d P(t)=\left(\frac{1}{\lambda}-\frac{1-\lambda}{\lambda} e^{\frac{t-T}{\delta^{2}}}\right) d t+\int_{\mathbf{E}} R(t, e) \tilde{N}(\text { dedt }), \quad t \in[s, T], \\
P(T)=0
\end{array}\right.
$$

which implies

$$
P(t)=\frac{1-\lambda}{\lambda} \delta^{2}\left(1-e^{\frac{s-T}{\delta^{2}}}\right)+\frac{1}{\lambda}(t-T), \quad \forall t \in[s, T] .
$$

Then the decreasing property of function $\rho(t) \equiv V_{x x}\left(t, \bar{x}^{s, y ; \bar{u}}(t)\right)+P(t)(\operatorname{noting} \rho(T)=0$ ) results in

$$
V_{x x}\left(t, \bar{x}^{s, y ; \bar{u}}(t)\right)=\delta^{2}\left(1-e^{\frac{t-T}{\delta^{2}}}\right)<-\frac{1-\lambda}{\lambda} \delta^{2}\left(1-e^{\frac{s-T}{\delta^{2}}}\right)-\frac{1}{\lambda}(t-T)=-P(t), \quad \forall t \in[0, T) .
$$

We proceed to study the super-subjets of the value function in the time variable $t$ along an optimal trajectory. Different from its deterministic counterpart (see [18]) and similar to but more complicated than its diffusion (without jumps) counterpart (see [18] or [19]), we observe that it is not the generalized Hamiltonian $G$ that is to be maximized in the stochastic MP unless $V$ is sufficiently smooth. Instead, it is the following $\mathcal{G}$-function which contains an additional term than the $\mathcal{H}$-function in the stochastic MP (Theorem 2.3). Associated with an optimal pair $\left(\bar{x}^{s, y ;} \bar{u}(\cdot), \bar{u}(\cdot)\right)$, its corresponding adjoint processes $(p(\cdot), q(\cdot), \gamma(\cdot, \cdot))$ and $(P(\cdot), Q(\cdot), R(\cdot, \cdot))$ satisfying (20), we define a $\mathcal{G}$-function $\mathcal{G}:[0, T] \times \mathbf{R}^{n} \times \mathbf{U} \rightarrow \mathbf{R}$ as 


$$
\begin{aligned}
& \mathcal{G}(t, x, u) \\
:= & \mathcal{H}(t, x, u)+\frac{1}{2} \operatorname{tr}\left\{R(t, e) \bar{c}(t, e) \bar{c}(t, e)^{\top}\right\} \pi(d e) \\
\equiv & \langle p(t), b(t, x, u)\rangle+\operatorname{tr}\left\{q(t)^{\top} \sigma(t, x, u)\right\}+\int_{\mathbf{E}}\langle\gamma(t, e), c(t, x, u, e)\rangle \pi(d e)-f(t, x, u) \\
& +\frac{1}{2} \operatorname{tr}\left\{P(t) \sigma(t, x, u) \sigma(t, x, u)^{\top}-2 P(t) \sigma(t, x, u) \bar{\sigma}(t)^{\top}\right\} \\
& +\frac{1}{2} \operatorname{tr}\left\{\int_{\mathbf{E}}\left[P(t) c(t, x, u, e) c(t, x, u, e)^{\top}-2 P(t) c(t, x, u, e) \bar{c}(t, e)^{\top}\right] \pi(d e)\right\} \\
& -\frac{1}{2} \operatorname{tr}\left\{\int_{\mathbf{E}}\left[R(t, e) c(t, x, u, e) c(t, x, u, e)^{\top}-2 R(t, e) c(t, x, u, e) \bar{c}(t, e)^{\top}\right] \pi(d e)\right\} .
\end{aligned}
$$

Remark 3.3 Recall definitions of the Hamiltonian function $H$ (17) and the generalized Hamiltonian function $G(16)$, we can easily verify that they have the following relations to the above $\mathcal{G}$-function. For a $\left.\Psi \in C\left([0, T] \times \mathbf{R}^{n}\right)\right)$, we have

$$
\begin{aligned}
\mathcal{G}(t, x, u):= & G(t, x, u, \Psi(t, x), p(t), P(t))+\operatorname{tr}\left\{\sigma(t, x, u)^{\top}[q(t)-P(t) \bar{\sigma}(t)]\right\} \\
& -\frac{1}{2} \operatorname{tr}\left\{P(t) \int_{\mathbf{E}}\left[\bar{c}(t, e) \bar{c}(t, e)^{\top}+\Delta c(t, e ; u) \Delta c(t, e ; u)^{\top}\right] \pi(d e)\right\} \\
& +\frac{1}{2} \operatorname{tr}\left\{\int_{\mathbf{E}} R(t, e) \Delta c(t, e ; u) \Delta c(t, e ; u)^{\top} \pi(d e)\right\} \\
& +\int_{\mathbf{E}}[\Psi(t, x+c(t, x, u, e))-\Psi(t, x)+\langle p(t)+\gamma(t, e), c(t, x, u, e)\rangle] \pi(d e) \\
= & H(t, x, u, p(t), q(t), \gamma(t, \cdot))-\frac{1}{2} \operatorname{tr}\left\{P(t)\left[\bar{\sigma}(t) \bar{\sigma}(t)^{\top}+\int_{\mathbf{E}} \bar{c}(t, e) \bar{c}(t, e)^{\top} \pi(d e)\right]\right\} \\
& +\frac{1}{2} \operatorname{tr}\left\{P(t)\left[\Delta \sigma(t ; u) \Delta \sigma(t ; u)^{\top}+\int_{\mathbf{E}} \Delta c(t, e ; u) \Delta c(t, e ; u)^{\top} \pi(d e)\right]\right\} \\
& +\frac{1}{2} \operatorname{tr}\left\{\int_{\mathbf{E}} R(t, e) \Delta c(t, e ; u) \Delta c(t, e ; u)^{\top} \pi(d e)\right\} .
\end{aligned}
$$

Note that, unlike the definition of generalized Hamiltonian function $G$, the $\mathcal{G}$-function can be defined associated with only $\left.\Psi \in C\left([0, T] \times \mathbf{R}^{n}\right)\right)$.

We first recall a few results on right Lesbesgue points for functions with values in abstract spaces (see also in pp. 2013-2014 of [8]).

Definition 3.3 Let $Z$ be a Banach space and let $z:[a, b] \rightarrow Z$ be a measurable function that is Bochner integrable. We say that $t$ is a right Lesbesgue point of $z$ if

$$
\lim _{h \rightarrow 0^{+}} \frac{1}{h} \int_{t}^{t+h}|z(r)-z(t)|_{z} d r=0
$$

Lemma 3.1 Let $z:[a, b] \rightarrow Z$ be as in Definition 3.3. Then the set of right Lesbesgue points of $z$ is of full measure in $[a, b]$. 
The second main result in this subsection is the following.

Theorem 3.3 Suppose that (H1) $\sim(\mathbf{H} 3)$ hold and let $(s, y) \in[0, T) \times \mathbf{R}^{n}$ be fixed. Let $\left(\bar{x}^{s, y ; \bar{u}}(\cdot), \bar{u}(\cdot)\right)$ be an optimal pair of our stochastic control problem. Let $(p(\cdot), q(\cdot), \gamma(\cdot, \cdot))$ and $(P(\cdot), Q(\cdot), R(\cdot, \cdot))$ are first-order and second-order adjoint processes, respectively. Then we have

$$
\mathcal{G}\left(t, \bar{x}^{s, y ; \bar{u}}(t), \bar{u}(t)\right) \in \mathcal{P}_{t+}^{1,+} V\left(t, \bar{x}^{s, y ; \bar{u}}(t)\right), \quad \text { a.e.t } \in[s, T], \mathbf{P} \text {-a.s., }
$$

where the $\mathcal{G}$-function is defined by (51).

Proof For any $t \in(s, T)$, take $\tau \in(t, T]$. Denote by $x_{\tau}(\cdot)$ the solution of the following SDEP

$$
\begin{aligned}
x_{\tau}(r)= & \bar{x}^{s, y ; \bar{u}}(t)+\int_{\tau}^{r} b\left(\theta, x_{\tau}(\theta), \bar{u}(\theta)\right) d \theta+\int_{\tau}^{r} \sigma\left(\theta, x_{\tau}(\theta), \bar{u}(\theta)\right) d W(\theta) \\
& +\int_{\mathbf{E}} \int_{\tau}^{r} c\left(\theta, x_{\tau}(\theta-), \bar{u}(\theta), e\right) \tilde{N}(\operatorname{ded} \theta), \quad r \in[\tau, T] .
\end{aligned}
$$

Set $\xi_{\tau}(r):=x_{\tau}(r)-\bar{x}^{s, y ; \bar{u}}(r), \tau \leq r \leq T$. Working under the new probability measure $\mathbf{P}\left(\cdot \mid \mathcal{F}_{\tau}^{S}\right)(\omega)$, we have the following estimate by $(10)$ :

$$
\mathbb{E}\left[\sup _{\tau \leq r \leq T}\left|\xi_{\tau}(r)\right|^{2} \mid \mathcal{F}_{\tau}^{s}\right](\omega) \leq C\left|\bar{x}^{s, y ; \bar{u}}(\tau, \omega)-\bar{x}^{s, y ; \bar{u}}(t, \omega)\right|^{2}, \quad \text { P-a.s. } \omega .
$$

Taking $\mathbb{E}\left(\cdot \mid \mathcal{F}_{t}^{S}\right)(\omega)$ on both sides and noting that $\mathcal{F}_{t}^{S} \subseteq \mathcal{F}_{\tau}^{s}$, by (11), we obtain

$$
\mathbb{E}\left[\sup _{\tau \leq r \leq T}\left|\xi_{\tau}(r)\right|^{2} \mid \mathcal{F}_{t}^{s}\right](\omega) \leq C|\tau-t|, \quad \text { P-a.s.w. }
$$

The process $\xi_{\tau}(\cdot)$ satisfies the following variational equations:

$$
\left\{\begin{aligned}
d \xi_{\tau}(r)= & \bar{b}_{x}(r) \xi_{\tau}(r) d r+\bar{\sigma}_{x}(r) \xi_{\tau}(r) d W(r)+\int_{\mathbf{E}} \bar{c}_{x}(r, e) \xi_{\tau}(r-) \tilde{N}(d e d r) \\
& +\varepsilon_{\tau 1}(r) d r+\varepsilon_{\tau 2}(r) d W(r)+\int_{\mathbf{E}} \varepsilon_{\tau 3}(r, e) \tilde{N}(d e d r), \quad r \in[t, T], \\
\xi_{\tau}(\tau)=- & \int_{t}^{\tau} \bar{b}(r) d r-\int_{t}^{\tau} \bar{\sigma}(r) d W(r)-\int_{\mathbf{E}} \int_{t}^{\tau} \bar{c}(r, e) \tilde{N}(d e d r)
\end{aligned}\right.
$$

and

$$
\left\{\begin{aligned}
d \xi_{\tau}(r)=\{ & \left.\bar{b}_{x}(r) \xi_{\tau}(r)+\frac{1}{2} \xi_{\tau}(r)^{\top} \bar{b}_{x x}(r) \xi_{\tau}(r)\right\} d r \\
& +\left\{\bar{\sigma}_{x}(r) \xi_{\tau}(r)+\frac{1}{2} \xi_{\tau}(r)^{\top} \bar{\sigma}_{x x}(r) \xi_{\tau}(r)\right\} d W(r) \\
& +\int_{\mathbf{E}}\left\{\bar{c}_{x}(r, e) \xi_{\tau}(r-)+\frac{1}{2} \xi_{\tau}(r-)^{\top} \bar{c}_{x x}(r, e) \xi_{\tau}(r-)\right\} \tilde{N}(d e d r) \\
& +\varepsilon_{\tau 4}(r) d r+\varepsilon_{\tau 5}(r) d W(r)+\int_{\mathbf{E}} \varepsilon_{\tau 6}(r, e) \tilde{N}(d e d r), \quad r \in[t, T] \\
\xi_{\tau}(\tau)=- & \int_{t}^{\tau} \bar{b}(r) d r-\int_{t}^{\tau} \bar{\sigma}(r) d W(r)-\int_{\mathbf{E}} \int_{t}^{\tau} \bar{c}(r, e) \tilde{N}(d e d r)
\end{aligned}\right.
$$


where

$$
\left\{\begin{aligned}
\varepsilon_{\tau 1}(r) & :=\int_{0}^{1}\left[b_{x}\left(r, \bar{x}^{s, y ; \bar{u}}(r)+\theta \xi_{\tau}(r), \bar{u}(r)\right)-\bar{b}_{x}(r)\right] \xi_{\tau}(r) d \theta, \\
\varepsilon_{\tau 2}(r) & :=\int_{0}^{1}\left[\sigma_{x}\left(r, \bar{x}^{s, y ; j \bar{u}}(r)+\theta \xi_{\tau}(r), \bar{u}(r)\right)-\bar{\sigma}_{x}(r)\right] \xi_{\tau}(r) d \theta, \\
\varepsilon_{\tau 3}(r, \cdot) & :=\int_{0}^{1}\left[c_{x}\left(r, \bar{x}^{s, y ; \bar{u}}(r-)+\theta \xi_{\tau}(r-), \bar{u}(r), \cdot\right)-\bar{c}_{x}(r, \cdot)\right] \xi_{\tau}(r-) d \theta, \\
\varepsilon_{\tau 4}(r) & :=\int_{0}^{1}(1-\theta) \xi_{\tau}(r)^{\top}\left[b_{x x}\left(r, \bar{x}^{s, y ; \bar{u}}(r)+\theta \xi_{\tau}(r), \bar{u}(r)\right)-\bar{b}_{x x}(r)\right] \xi_{\tau}(r) d \theta, \\
\varepsilon_{\tau 5}(r) & :=\int_{0}^{1}(1-\theta) \xi_{\tau}(r)^{\top}\left[\sigma_{x x}\left(r, \bar{x}^{s, y ;} \bar{u}(r)+\theta \xi_{\tau}(r), \bar{u}(r)\right)-\bar{\sigma}_{x x}(r)\right] \xi_{\tau}(r) d \theta, \\
\varepsilon_{\tau 6}(r, \cdot) & :=\int_{0}^{1}(1-\theta) \xi_{\tau}(r-)^{\top}\left[c_{x x}\left(r, \bar{x}^{s, y ;}, \bar{u}(r-)+\theta \xi_{\tau}(r-), \bar{u}(r), \cdot\right)-\bar{c}_{x x}(r, \cdot)\right] \xi_{\tau}(r-) d \theta .
\end{aligned}\right.
$$

Similar to the proof of (41) and (42), there exists a deterministic continuous and increasing function $\delta:[0, \infty) \rightarrow[0, \infty)$, with $\frac{\delta(r)}{r} \rightarrow 0$ as $r \rightarrow 0$, such that

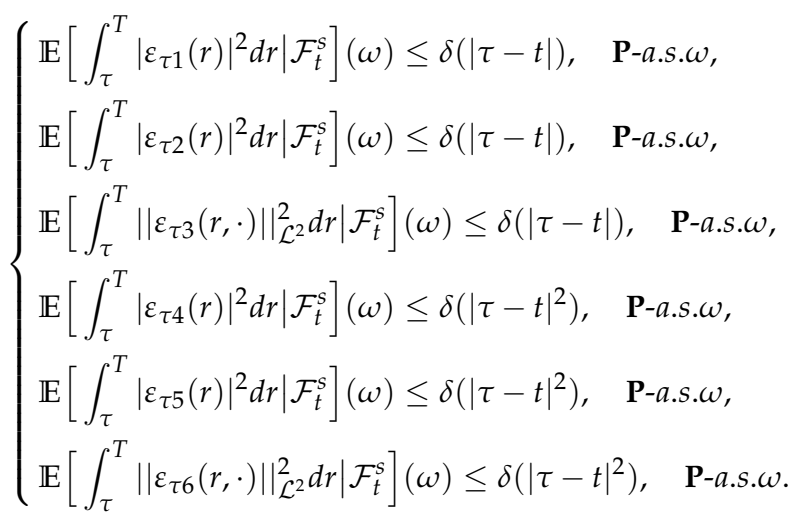

Note that $\left.\bar{u}(\cdot)\right|_{[\tau, T]} \in \mathcal{U}[\tau, T], \mathbf{P}$-a.s. Thus by the definition of the value function $V$, we have

$$
V\left(\tau, \bar{x}^{s, y ; \bar{u}}(t)\right) \leq \mathbb{E}\left[\int_{\tau}^{T} f\left(r, x_{\tau}(r), \bar{u}(r)\right) d r+h\left(x_{\tau}(T)\right) \mid \mathcal{F}_{\tau}^{s}\right], \quad \forall \tau \in(t, T], \mathbf{P}-a . s .
$$

Taking $\mathbb{E}\left(\cdot \mid \mathcal{F}_{t}^{S}\right)$ on both sides and noting that $\mathcal{F}_{t}^{S} \subseteq \mathcal{F}_{\tau}^{S}$, we conclude that

$$
V\left(\tau, \bar{x}^{s, y ; \bar{u}}(t)\right) \leq \mathbb{E}\left[\int_{\tau}^{T} f\left(r, x_{\tau}(r), \bar{u}(r)\right) d r+h\left(x_{\tau}(T)\right) \mid \mathcal{F}_{t}^{s}\right], \quad \forall \tau \in(t, T], \mathbf{P}-a . s .
$$

Choose a common subset $\Omega_{0} \subseteq \Omega$ with $P\left(\Omega_{0}\right)=1$ such that for any $\omega_{0} \in \Omega_{0}$

$$
\left\{\begin{array}{l}
V\left(t, \bar{x}^{s, y ; \bar{u}}\left(t, \omega_{0}\right)\right)=\mathbb{E}\left[\int_{\mathcal{s}}^{T} \bar{f}(r) d r+h\left(\bar{x}^{s, y ; \bar{u}}(T)\right) \mid \mathcal{F}_{t}^{s}\right]\left(\omega_{0}\right), \\
(54),(57) \text { are satisfied for any rational } \tau>t, \text { and }\left.\bar{u}(\cdot)\right|_{[\tau, T]} \in \mathcal{U}[\tau, T] .
\end{array}\right.
$$


Let $\omega_{0} \in \Omega_{0}$ be fixed, then for any rational $\tau>t$, we have (noting (58))

$$
\begin{aligned}
& V\left(\tau, \bar{x}^{s, y ; \bar{u}}\left(t, \omega_{0}\right)\right)-V\left(t, \bar{x}^{s, y ; \bar{u}}\left(t, \omega_{0}\right)\right) \\
\leq & \mathbb{E}\left\{-\int_{t}^{\tau} \bar{f}(r) d r+\int_{\tau}^{T}\left[f\left(r, x_{\tau}(r), \bar{u}(r)\right)-\bar{f}(r)\right] d r+h\left(x_{\tau}(T)\right)-h\left(\bar{x}^{s, y ; \bar{u}}(T)\right) \mid \mathcal{F}_{t}^{s}\right\}\left(\omega_{0}\right) \\
= & \mathbb{E}\left\{-\int_{t}^{\tau} \bar{f}(r) d r+\int_{\tau}^{T}\left\langle\bar{f}_{x}(r), \xi_{\tau}(r)\right\rangle d r+\left\langle h_{x}\left(\bar{x}^{s, y ; \bar{u}}(T)\right), \xi_{\tau}(T)\right\rangle\right. \\
& \left.+\frac{1}{2} \int_{\tau}^{T} \xi_{\tau}(r)^{\top} \bar{f}_{x x}(r) \xi_{\tau}(r) d r+\frac{1}{2} \xi_{\tau}(T)^{\top} h_{x x}\left(\bar{x}^{s, y ; \bar{u}}(T)\right) \xi_{\tau}(T) \mid \mathcal{F}_{t}^{s}\right\}\left(\omega_{0}\right)+o(|\tau-t|) .
\end{aligned}
$$

As in (48) (using the duality technique), we have

$$
\begin{aligned}
& V\left(\tau, \bar{x}^{s, y ; \bar{u}}\left(t, \omega_{0}\right)\right)-V\left(t, \bar{x}^{s, y ; \bar{u}}\left(t, \omega_{0}\right)\right) \\
\leq & -\mathbb{E}\left\{-\int_{t}^{\tau} \bar{f}(r) d r \mid \mathcal{F}_{t}^{s}\right\}\left(\omega_{0}\right)-\mathbb{E}\left\{\left\langle p(\tau), \xi_{\tau}(\tau)\right\rangle+\frac{1}{2} \xi_{\tau}(\tau)^{\top} P(\tau) \xi_{\tau}(\tau) \mid \mathcal{F}_{t}^{s}\right\}\left(\omega_{0}\right) \\
& +o(|\tau-t|) .
\end{aligned}
$$

Now let us estimate the terms on the right-hand side of (59). To this end, we first note that for any $\left.\varphi(\cdot), \hat{\varphi}(\cdot) \in L_{\mathcal{F}}^{2}\left([0, T] ; \mathbf{R}^{n}\right), \psi(\cdot) \in L_{\mathcal{F}, p}^{2}\left([0, T] ; \mathbf{R}^{n \times d}\right), \Phi(\cdot, \cdot)\right) \in F_{p}^{2}\left([0, T] ; \mathbf{R}^{n}\right)$, we have the following three estimates:

$$
\begin{gathered}
\mathbb{E}\left\{\left\langle\int_{t}^{\tau} \varphi(r) d r, \int_{t}^{\tau} \hat{\varphi}(r) d r\right\rangle \mid \mathcal{F}_{t}^{s}\right\}\left(\omega_{0}\right) \\
\leq\left\{\mathbb{E}\left[\left|\int_{t}^{\tau} \varphi(r) d r\right|^{2} \mid \mathcal{F}_{t}^{s}\right]\left(\omega_{0}\right)\right\}^{\frac{1}{2}}\left\{\mathbb{E}\left[\left|\int_{t}^{\tau} \hat{\varphi}(r) d r\right|^{2} \mid \mathcal{F}_{t}^{s}\right]\left(\omega_{0}\right)\right\}^{\frac{1}{2}} \\
\leq(\tau-t)\left\{\int_{t}^{\tau} \mathbb{E}\left[|\varphi(r)|^{2} \mid \mathcal{F}_{t}^{s}\right]\left(\omega_{0}\right) d r \int_{t}^{\tau} \mathbb{E}\left[|\hat{\varphi}(r)|^{2} \mid \mathcal{F}_{t}^{s}\right]\left(\omega_{0}\right) d r\right\}^{\frac{1}{2}} \\
=o(|\tau-t|), \quad \text { as } \tau \downarrow t, \quad \forall t \in[s, T), \\
\mathbb{E}\left\{\left\langle\int_{t}^{\tau} \varphi(r) d r, \int_{t}^{\tau} \psi(r) d W(r)\right\rangle \mid \mathcal{F}_{t}^{s}\right\}\left(\omega_{0}\right) \\
\leq\left\{\mathbb{E}\left[\left|\int_{t}^{\tau} \varphi(r) d r\right|^{2} \mid \mathcal{F}_{t}^{s}\right]\left(\omega_{0}\right)\right\}^{\frac{1}{2}}\left\{\mathbb{E}\left[\left|\int_{t}^{\tau} \psi(r) d W(r)\right|^{2} \mid \mathcal{F}_{t}^{s}\right]\left(\omega_{0}\right)\right\}^{\frac{1}{2}} \\
\leq(\tau-t)^{\frac{1}{2}}\left\{\int_{t}^{\tau} \mathbb{E}\left[|\varphi(r)|^{2} \mid \mathcal{F}_{t}^{s}\right]\left(\omega_{0}\right) d r \int_{t}^{\tau} \mathbb{E}\left[|\psi(r)|^{2} \mid \mathcal{F}_{t}^{s}\right]\left(\omega_{0}\right) d r\right\}^{\frac{1}{2}} \\
=o(|\tau-t|), \quad \text { as } \tau \downarrow t, \quad a . e . t \in[s, T), \\
\mathbb{E}\left\{\left\langle\int_{t}^{\tau} \varphi(r) d r, \int_{\mathbb{E}} \int_{t}^{\tau} \Phi(r, e) \tilde{N}(\text { dedr })\right\rangle \mid \mathcal{F}_{t}^{s}\right\}\left(\omega_{0}\right) \\
\leq\left\{\mathbb{E}\left[\left|\int_{t}^{\tau} \varphi(r) d r\right|^{2} \mid \mathcal{F}_{t}^{s}\right]\left(\omega_{0}\right)\right\}^{\frac{1}{2}}\left\{\mathbb{E}\left[\left|\int_{\mathbb{E}} \int_{t}^{\tau} \Phi(r, e) \tilde{N}(d e d r)\right|^{2} \mid \mathcal{F}_{t}^{s}\right]\left(\omega_{0}\right)\right\}^{\frac{1}{2}} \\
\leq(\tau-t)^{\frac{1}{2}}\left\{\int_{t}^{\tau} \mathbb{E}\left[|\varphi(r)|^{2} \mid \mathcal{F}_{t}^{s}\right]\left(\omega_{0}\right) d r \int_{\mathbb{E}} \int_{t}^{\tau} \mathbb{E}\left[|\Phi(r, e)|^{2} \mid \mathcal{F}_{t}^{s}\right]\left(\omega_{0}\right) \pi(d e) d r\right\}^{\frac{1}{2}} \\
=o(|\tau-t|), \text { as } \tau \downarrow t, \quad a . e . t \in[s, T) .
\end{gathered}
$$


All the last equalities in the above three inequalities is due to the fact that the sets of right Lebesgue points have full Lebesgue measures for integrable functions by Lemma 3.1 and $t \mapsto$ $\mathcal{F}_{t}^{s}$ is right continuous in $t$. Thus applying Itô's formula to $\left\langle p(\cdot), \xi_{\tau}(\cdot)\right\rangle$, by (55), (18) we have

$$
\begin{aligned}
\mathbb{E}\left\{\left\langle p(\tau), \xi_{\tau}(\tau)\right\rangle \mid \mathcal{F}_{t}^{s}\right\}\left(\omega_{0}\right) \\
=\mathbb{E}\left\{\left\langle p(t), \xi_{\tau}(\tau)\right\rangle+\left\langle p(\tau)-p(t), \xi_{\tau}(\tau)\right\rangle \mid \mathcal{F}_{t}^{s}\right\}\left(\omega_{0}\right) \\
=\mathbb{E}\left\{\left\langle p(t),-\int_{t}^{\tau} \bar{b}(r) d r-\int_{t}^{\tau} \bar{\sigma}(r) d W(r)-\int_{\mathbf{E}} \int_{t}^{\tau} \bar{c}(r, e) \tilde{N}(d e d r)\right\rangle\right. \\
+\left\langle-\int_{t}^{\tau}\left(\bar{b}_{x}(r) p(r)+\bar{\sigma}_{x}(r) q(r)+\int_{\mathbf{E}} \bar{c}_{x}(r, e) \gamma(r, e) \pi(d e)\right) d r\right. \\
\quad+\int_{t}^{\tau} q(r) d W(r)+\int_{\mathbf{E}} \int_{t}^{\tau} \gamma(r, e) \tilde{N}(d e d r),-\int_{t}^{\tau} \bar{b}(r) d r \\
\left.\left.\quad-\int_{t}^{\tau} \bar{\sigma}(r) d W(r)-\int_{\mathbf{E}} \int_{t}^{\tau} \bar{c}(r, e) \tilde{N}(d e d r)\right\rangle \mid \mathcal{F}_{t}^{s}\right\}\left(\omega_{0}\right) \\
=\mathbb{E}\left\{-\left\langle p(t), \int_{t}^{\tau} \bar{b}(r) d r\right\rangle-\int_{t}^{\tau} \operatorname{tr}\left\{q(r)^{\top} \bar{\sigma}(r)\right\} d r\right. \\
\left.\quad-\int_{\mathbf{E}} \int_{t}^{\tau}\langle\gamma(r, e), \bar{c}(r, e)\rangle \pi(d e) d r \mid \mathcal{F}_{t}^{s}\right\}\left(\omega_{0}\right)+o(|\tau-t|), \quad \text { as } \tau \downarrow t \text {, a.e.t } \in[s, T) .
\end{aligned}
$$

Similarly, applying Itô's formula to $\left.\xi_{\tau}(\cdot)^{\top} P(\cdot) \xi_{\tau}(\cdot)\right\rangle$, by (56) and (19), we have

$$
\begin{aligned}
& \mathbb{E}\left\{\xi_{\tau}(\tau)^{\top} P(\tau) \xi_{\tau}(\tau) \mid \mathcal{F}_{t}^{s}\right\}\left(\omega_{0}\right) \\
= & \mathbb{E}\left\{\int_{t}^{\tau} \operatorname{tr}\left\{\bar{\sigma}(r)^{\top} P(t) \bar{\sigma}(r)\right\} d r+\int_{\mathbf{E}} \int_{t}^{\tau} \operatorname{tr}\left\{\bar{c}(r, e)^{\top} P(t) \bar{c}(r, e)\right\} \pi(d e) d r \mid \mathcal{F}_{t}^{s}\right\}\left(\omega_{0}\right) \\
& +o(|\tau-t|), \quad \text { as } \tau \downarrow t, \quad \text { a.e.t } \in[s, T) .
\end{aligned}
$$

It follows from (59), (60) and (61) that for any rational $\tau \in(t, T]$,

$$
\begin{aligned}
V\left(\tau, \bar{x}^{s, y ; \bar{u}}\left(t, \omega_{0}\right)\right)-V\left(t, \bar{x}^{s, y ;} ; \bar{u}\left(t, \omega_{0}\right)\right) \\
\leq \mathbb{E}\left\{-\int_{t}^{\tau} \bar{f}(r) d r+\left\langle p(t), \int_{t}^{\tau} \bar{b}(r) d r\right\rangle+\int_{t}^{\tau} \operatorname{tr}\left\{q(r)^{\top} \bar{\sigma}(r)\right\} d r\right. \\
\quad-\frac{1}{2} \int_{t}^{\tau} \operatorname{tr}\left[\bar{\sigma}(r)^{\top} P(t) \bar{\sigma}(r)\right] d r+\int_{\mathbf{E}} \int_{t}^{\tau}\left\langle\gamma(r, e)^{\top} \bar{c}(r, e)\right\rangle \pi(d e) d r \\
\left.\quad-\frac{1}{2} \int_{\mathbf{E}} \int_{t}^{\tau} \operatorname{tr}\left\{\bar{c}(r, e)^{\top} P(t) \bar{c}(r, e)\right\} \pi(d e) d r \mid \mathcal{F}_{t}^{s}\right\}\left(\omega_{0}\right)+o(|\tau-t|) \\
\leq(\tau-t)\left\{\mathcal{H}\left(t, \bar{x}^{s, y ; \bar{u}}\left(t, \omega_{0}\right), \bar{u}(t)\right)+\frac{1}{2} \operatorname{tr}\left\{R(t, e) \bar{c}(t, e) \bar{c}(t, e)^{\top}\right\} \pi(d e)\right\} \\
=(\tau-t) \mathcal{G}\left(t, \bar{x}^{s, y ; \bar{u}}\left(t, \omega_{0}\right), \bar{u}(t)\right)+o(|\tau-t|), \quad \text { as } \tau \downarrow t, \quad \text { a.e.t } \in[s, T) .
\end{aligned}
$$

By the same argument as in the paragraph following (49), we conclude that (62) holds for any $\tau>t$. By definition (30), then (52) holds. The proof is complete.

Remark 3.4 As aforementioned, it is worth to point out that by comparing the result of Theorem 3.3 with those analogue in the diffusion case (see Theorem 4.7 on pp. 263 of [18]), we observe that an additional term $-\frac{1}{2} \operatorname{tr}\left\{R(t, e) \bar{c}(t, e) \bar{c}(t, e)^{\top}\right\} \pi(d e)$ - containing the integrand 
with respect to the compensated martingale measure $\tilde{N}$ in the second-order adjoint equation appears in the $\mathcal{G}$-function of (52). This is different from the continuous diffusion case where this $\mathcal{G}$-function coincides with the $\mathcal{H}$-function appearing in the maximum principle.

Now, let us combine Theorem 3.2 and 3.3 to get the following result.

Theorem 3.4 Suppose that (H1) $\sim(\mathbf{H} 3)$ hold and let $(s, y) \in[0, T) \times \mathbf{R}^{n}$ be fixed. Let $\left(\bar{x}^{s, y ;} ; \bar{u}(\cdot), \bar{u}(\cdot)\right)$ be the optimal pair of our stochastic control problem. Let $(p(\cdot), q(\cdot), \gamma(\cdot, \cdot))$ and $(P(\cdot), Q(\cdot), R(\cdot, \cdot))$ are first-order and second-order adjoint processes, respectively. Then we have

$$
\begin{gathered}
{\left[\mathcal{G}\left(t, \bar{x}^{s, y ; \bar{u}}(t), \bar{u}(t)\right), \infty\right) \times\{-p(t)\} \times[-P(t), \infty) \subseteq \mathcal{P}_{t+, x}^{1,2,+} V\left(t, \bar{x}^{s, y ; \bar{u}}(t)\right), \text { a.e.t } \in[s, T], \mathbf{P} \text {-a.s., }} \\
\mathcal{P}_{t+, x}^{1,2,-} V\left(t, \bar{x}^{s, y ; \bar{u}}(t)\right) \subseteq\left(-\infty, \mathcal{G}\left(t, \bar{x}^{s, y ; \bar{u}}(t), \bar{u}(t)\right)\right] \times\{-p(t)\} \times(-\infty, P(t)], \text { a.e.t } \in[s, T], \mathbf{P}-\text { a.s. }
\end{gathered}
$$

Proof The first conclusion (63) can be proved by combining the proofs of (34) and (52) and making use of (3.1). We now show (64). For any $q \in \mathcal{P}_{t+}^{1,-} V\left(t, \bar{x}^{s, y ; i \bar{u}}(t)\right)$, by definition (31) and (62) we have

$$
\begin{aligned}
0 & \leq V\left(\tau, \bar{x}^{s, y ; \bar{u}}(t)\right)-V\left(t, \bar{x}^{s, y ; \bar{u}}(t)\right)-q(\tau-t) \\
& =\left(\mathcal{G}\left(t, \bar{x}^{s, y ;} ; \bar{u}(t), \bar{u}(t)\right)-q\right)(\tau-t)+o(|\tau-t|), \quad \text { a.e.t } \in[s, T], \mathbf{P}-a . s .
\end{aligned}
$$

Then it is necessary that

$$
q \leq \mathcal{G}\left(t, \bar{x}^{s, y ; \bar{u}}(t), \bar{u}(t)\right), \quad \text { a.e.t } \in[s, T], \mathbf{P}-\text { a.s. }
$$

From this and (35), we have (64). The proof is complete.

Theorem 3.4 is a generalization of the classical result on the relationship between stochastic MP and DPP (see Theorem 2.1 of [7]). On the other hand, we do have a simple example showing that both the set inclusions in (63) and (64) may be strict.

Example 3.2 Consider the following linear stochastic control system with Poisson jumps $(n=d=1)$ :

$$
\left\{\begin{aligned}
d x^{s, y ; u}(t) & =x^{s, y ; u}(t) u(t) d t+x^{s, y ; u}(t) d W(t)+x^{s, y ; u}(t-) u(t) \tilde{N}(d t), \quad t \in[s, T], \\
x^{s, y ; u}(s) & =y .
\end{aligned}\right.
$$

Here $N$ is a Poisson process with the intensity $\lambda d t$ and $\tilde{N}(d t):=N(d t)-\lambda d t(\lambda>0)$ is the compensated martingale measure. The control domain is $\mathbf{U}=[0,1]$ and the cost functional is

$$
J(s, y ; u(\cdot))=-\mathbb{E} x^{s, y ; u}(T) .
$$

The corresponding HJB equation (15) now reads

$$
\left\{\begin{array}{l}
-v_{t}(t, x)-\lambda v(t, x)-\frac{1}{2} x^{2} v_{x x}(t, x) \\
\quad+\sup _{0 \leq u \leq 1}\left[(1-\lambda) v_{x}(t, x) x u+\lambda v(t, x+x u)\right]=0, \quad t \in[s, T], \\
v(T, x)=-x .
\end{array}\right.
$$


It is not difficulty to verify that the following function is a viscosity solution of (3.2):

$$
V(t, x)= \begin{cases}-x, & \text { if } x \leq 0, \\ -x e^{T-t}, & \text { if } x>0,\end{cases}
$$

which clearly satisfy (12) and (13). Thus, by the uniqueness of the viscosity solution, $V$ coincides with the value function of the optimal control problem. However, it is not in $C^{1,2}([0, T] \times \mathbf{R})$, since $V_{x}(t, x)$ has a jump at $(t, 0)$ for all $0 \leq t<T$. On the other hand, we have (noting (18))

$$
\left\{\begin{aligned}
-d p(t) & =[\bar{u}(t) p(t)+q(t)+\lambda \gamma(t) \bar{u}(t)] d t-q(t) d W(t)-\gamma(t) \tilde{N}(d t), \quad t \in[s, T], \\
p(T) & =1 .
\end{aligned}\right.
$$

We can solve that

$$
(p(t), q(t), \gamma(t))=\left(e^{\int_{t}^{T} \bar{u}(s) d s}, 0,0\right)
$$

Then with

$$
\mathcal{H}\left(t, \bar{x}^{s, y ; \bar{u}}(t), u\right)=e^{\int_{t}^{T} \bar{u}(s) d s} \bar{x}^{s, y ; \bar{u}}(t) u-\lambda \bar{u}(t) \bar{x}^{s, y ; \bar{u}}(t)^{2} u+\frac{1}{2} \lambda \bar{x}^{s, y ; \bar{u}}(t)^{2} u^{2},
$$

by Theorem 2.3, we have for $t \in[s, T]$,

$$
\bar{u}(t)= \begin{cases}1, & \bar{x}^{s, y ; \bar{u}}(t)>0, \\ 0, & \bar{x}^{s, y ; \bar{u}}(t) \leq 0 .\end{cases}
$$

Now consider $s=0, y=0$. Clearly, $\left(\bar{x}^{0,0 ; \bar{u}}(\cdot), \bar{u}(\cdot)\right) \equiv(0,0)$ is an optimal control. Theorem 2.1 of [7] does not apply, because $V_{x}(t, x)$ does not exist along the whole trajectory $\bar{x}^{0,0 ; \bar{u}}(t), t \in$ $[0, T]$. However, one can show that

$$
\left\{\begin{array}{l}
\mathcal{P}_{t+, x}^{1,2,+} V\left(t, \bar{x}^{0,0 ; \bar{u}}(t)\right)=\mathcal{P}_{t+, x}^{1,2,+} V(t, 0)=[0, \infty) \times\left[-e^{T-t},-1\right] \times[0, \infty), \quad t \in[0, T], \\
\mathcal{P}_{t+, x}^{1,2,-} V\left(t, \bar{x}^{0,0 ; \bar{u}}(t)\right)=\mathcal{P}_{t+, x}^{1,2,-} V(t, 0)=[-\infty, 0] \times \varnothing \times \varnothing, \quad t \in[0, T], \\
p(t)=1, \quad t \in[0, T], \quad P(t)=0, \quad t \in[0, T], \\
\mathcal{G}\left(t, \bar{x}^{0,0 ; \bar{u}}(t), \bar{u}(t)\right)=\mathcal{G}(t, 0,0)=0, \quad t \in[0, T] .
\end{array}\right.
$$

Thus, it is clear that both the set inclusions in (63) and (64) of Theorem 3.4 are strict for $t \in$ $[0, T)$.

The following result is the special case when we assume the value function is enough smooth.

Corollary 3.1 Suppose that (H1) $\sim(\mathbf{H} 3)$ hold and let $(s, y) \in[0, T) \times \mathbf{R}^{n}$ be fixed. Let $\left(\bar{x}^{s, y ; \bar{u}}(\cdot), \bar{u}(\cdot)\right)$ be an optimal pair of our stochastic optimal control problem and $(p(\cdot), q(\cdot), \gamma(\cdot, \cdot))$ be the first-order adjoint processes. Suppose the value function $V \in C^{1,2}\left([0, T] \times \mathbf{R}^{n}\right)$. Then

$$
\begin{array}{r}
V_{t}\left(t, \bar{x}^{s, y ; \bar{u}}(t)\right)=G\left(t, \bar{x}^{s, y ; \bar{u}}(t), \bar{u}(t),-V\left(t, \bar{x}^{s, y ; \bar{u}}(t)\right),-V_{x}\left(t, \bar{x}^{s, y ; \bar{u}}(t)\right),-V_{x x}\left(t, \bar{x}^{s, y ; \bar{u}}(t)\right)\right) \\
=\sup _{u \in \mathbf{U}} G\left(t, \bar{x}^{s, y ; \bar{u}}(t), u,-V\left(t, \bar{x}^{s, y ; \bar{u}}(t)\right),-V_{x}\left(t, \bar{x}^{s, y ;} ; \bar{u}(t)\right),-V_{x x}\left(t, \bar{x}^{s, y ; \bar{u}}(t)\right)\right), \\
\text { a.e.t } \in[s, T], \mathbf{P}-\text { a.s., }
\end{array}
$$


where $G$ is defined by (16). Moreover, if $V \in C^{1,3}\left([0, T] \times \mathbf{R}^{n}\right)$ and $V_{t x}$ is also continuous, then

$$
\left\{\begin{array}{l}
V_{x}\left(t, \bar{x}^{s, y ; \bar{u}}(t)\right)=-p(t), \quad \forall t \in[s, T], \mathbf{P}-a . s ., \\
V_{x x}\left(t, \bar{x}^{s, y ; \bar{u}}(t)\right) \bar{\sigma}(t)=-q(t), \quad \text { a.e.t } \in[s, T], \mathbf{P}-a . s ., \\
V_{x}\left(t, \bar{x}^{s, y ; \bar{u}}(t-)+\bar{c}(t, \cdot)\right)-V_{x}\left(t, \bar{x}^{s, y ; \bar{u}}(t-)\right)=-\gamma(t, \cdot), \quad \text { a.e.t } \in[s, T], \mathbf{P} \text {-a.s. }
\end{array}\right.
$$

By martingale representation theorem (see Lemma 2.3, [16]) and Itô's formula (see [10]), the proof technique is quite similar to Theorem 4.1, Chapter 4 of [18]. So we omit the detail. In fact, the relationship in (67) also can be seen in Theorem 2.1 of [7]. See also (3) in Introduction of this chapter.

Remark 3.5 (i) On the assumption that the value function $V$ is smooth, the first equality in (66) show us the relationship between the derivative of $V$ with respective to the time variable and the generalized Hamiltonian function $G$ defined by (16) along an optimal state trajectory.

(ii) It is interesting to note that the second equality in (66) may be regard as a "maximum principle" in terms of the value function and its derivatives. It is different from the stochastic MP aforementioned (Theorem 2.3), where no value function or its derivatives is involved.

(iii) The three equalities in (67) show us the relationship between the derivative of $V$ with respective to the state variable and the adjoint processes $p, q, \gamma(\cdot)$. More precisely, the three adjoint processes $p, q, \gamma(\cdot)$ can be expressed in terms of the derivatives of $V$ with respective to the state variable along an optimal state trajectory. It is also interesting to note that from the third equality in (67), we observe that the jump amplitude of $V_{x}\left(t, \bar{x}^{s, y ; i}(t)\right)$ equal to $-\gamma(t, \cdot)$ which is just that $V_{x}\left(t, \bar{x}^{s, y ; \bar{u}}(t)\right)=-p(t)$ tell us by the first-order adjoint equation (18).

Remark 3.6 By Remark 3.2 and Example 3.1, it can be seen that though the first classical relation in (67) of Corollary 3.1 is recovered from Theorem 3.2 when the value function $V$ is smooth enough, the nonsmooth version of the second classical relation in (67), i.e., $q(t)=$ $P(t) \bar{\sigma}(t)$, does not hold in general. We are also interested to the question that to what extent the third classical relation in (67) can be generalized when $V$ is not smooth. However, it seems that Theorem 3.2 tells us nothing in this context while the following result gives the general relationship among $p, q, \gamma(\cdot), P, \bar{\sigma}$ and $\bar{c}(\cdot)$.

Proposition 3.3 Under the assumption of Theorem 3.4, we have

$$
\begin{aligned}
0 \leq & \operatorname{tr}\left\{\bar{\sigma}(t)^{\top}[q(t)-P(t) \bar{\sigma}(t)]\right\}-\frac{1}{2} \operatorname{tr}\left\{P(t) \int_{\mathbf{E}} \bar{c}(t, e) \bar{c}(t, e)^{\top} \pi(d e)\right\} \\
& +\int_{\mathbf{E}}\left[\psi_{1}(t, x+\bar{c}(t, e))-\psi_{1}(t, x)+\langle p(t)+\gamma(t, e), \bar{c}(t, e)\rangle\right] \pi(\text { de }), \quad \text { a.e.t } \in[s, T], \mathbf{P} \text {-a.s., }
\end{aligned}
$$

or, equivalently,

$$
\mathcal{G}\left(t, \bar{x}^{s, y ; \bar{u}}(t), \bar{u}(t)\right) \geq G\left(t, \bar{x}^{s, y ; \bar{u}}(t), \bar{u}(t),-\psi_{1}\left(t, \bar{x}^{s, y ; \bar{u}}(t)\right), p(t), P(t)\right), \quad \text { a.e.t } \in[s, T], \mathbf{P}-a . s .,
$$

where $\psi_{1} \in C^{1,2}\left([0, T] \times \mathbf{R}^{n}\right)$, such that $\psi_{1}\left(t^{\prime}, x^{\prime}\right)>V\left(t^{\prime}, x^{\prime}\right), \forall\left(t^{\prime}, x^{\prime}\right) \neq(t, x) \in[s, T] \times \mathbf{R}^{n}$. 
Proof By (63) and the fact that $V$ is a viscosity solution of (15), we have

$$
\begin{aligned}
0 \geq & -\mathcal{G}\left(t, \bar{x}^{s, y ; \bar{u}}(t), \bar{u}(t)\right)+\sup _{u \in \mathbf{U}} G\left(t, \bar{x}^{s, y ; i \bar{u}}(t), u,-\psi_{1}\left(t, \bar{x}^{s, y ; j}(t)\right), p(t), P(t)\right) \\
\geq & -G\left(t, \bar{x}^{s, y ; j \bar{u}}(t), \bar{u}(t),-\psi_{1}\left(t, \bar{x}^{s, y ; \bar{u}}(t)\right), p(t), P(t)\right) \\
& +\sup _{u \in \mathbf{U}} G\left(t, \bar{x}^{s, y ;} \bar{u}(t), u,-\psi_{1}\left(t, \bar{x}^{s, y ;} ; \bar{u}(t)\right), p(t), P(t)\right) \\
& -\operatorname{tr}\left\{\bar{\sigma}(t)^{\top}[q(t)-P(t) \bar{\sigma}(t)]\right\}+\frac{1}{2} \operatorname{tr}\left\{P(t) \int_{\mathbf{E}} \bar{c}(t, e) \bar{c}(t, e)^{\top} \pi(d e)\right\} \\
& -\int_{\mathbf{E}}\left[\psi_{1}(t, x+\bar{c}(t, e))-\psi_{1}(t, x)+\langle p(t)+\gamma(t, e), \bar{c}(t, e)\rangle\right] \pi(d e) .
\end{aligned}
$$

Then, (68) or (69) follows. The proof is complete.

\section{Conclusion}

In this chapter, we have derived the relationship between the maximum principle and dynamic programming principle for the stochastic optimal control problem of jump diffusions. Without involving any derivatives of the value function, relations among the adjoint processes, the generalized Hamiltonian and the value function are derived in the language of viscosity solutions and the associated super-subjets. The conditions under which the above results are valid are very mild and reasonable. The results in this chapter bridge an important gap in the literature.

\section{Author details}

Jingtao Shi

Shandong University, P. R. China

\section{References}

[1] Alvarez, D. \& Tourin, A. (1996). Viscosity solutions of nonlinear integral-differential equations. Ann. Inst. H. Poincaré Anal. Non Linéaire, Vol. 13, No. 3, 293-317.

[2] Barles, G., Buckdahn, R. \& Pardoux, E. (1997). Backward stochastic differential equations and integral-partial differential equations. Stochastics $\mathcal{E}$ Stochastics Reports, Vol. 60, 57-83.

[3] Barles, G. \& Imbert, C. (2008). Second-order elliptic integral-differential equations: Viscosity solutions' theory revisited. Ann. Inst. H. Poincaré Anal. Non Linéaire, Vol. 25, No. 3, 567-585.

[4] Bensoussan, A. (1981). Lectures on stochastic control. Lecture Notes in Mathematics, Vol. 972, Springer-Verlag, Berlin.

[5] Bismut, J.M. (1978). An introductory approach to duality in optimal stochastic control. SIAM Journal on Control and Optimization, Vol. 20, No. 1, 62-78.

[6] Biswas, I.H., Jakobsen, E.R. \& Karlsen, K.H. (2010). Viscosity solutions for a system of integro-PDEs and connections to optimal switching and control of jump-diffusion processes. Applied Mathematics and Optimization, Vol. 62, No. 1, 47-80. 
[7] Framstad, N.C., Øksendal, B. \& Sulem, A. (2004). A sufficient stochastic maximum principle for optimal control of jump diffusions and applications to finance. Journal of Optimization Theory and Applications, Vol. 121, No. 1, 77-98. (Errata (2005), this journal, Vol. 124, No. 2, 511-512.)

[8] Gozzi, F., Swiech, A. \& Zhou, X.Y. (2005). A corrected proof of the stochastic verification theorem within the framework of viscosity solutions. SIAM Journal on Control and Optimization, Vol. 43, No. 6, 2009-2019.

[9] Haussmann, U.G. (1981). On the adjoint process for optimal control of diffusion processes. SIAM Journal on Control and Optimization, Vol. 19, No. 2, 221-243.

[10] Ikeda, N. \& Watanabe, S. (1989). Stochastic Differential Equations and Diffusion Processes. North-Holland, Kodansha.

[11] Li, J. \& Peng, S.G. (2009). Stochastic optimization theory of backward stochastic differential equations with jumps and viscosity solutions of Hamilton-Jacobi-Bellman equations. Nonlinear Analysis, Vol. 70, 1776-1796.

[12] Øksendal, B. \& Sulem, A. (2005). Applied Stochastic Control of Jump Diffusions. Springer-Verlag, Berlin.

[13] Pardoux, E. \& Peng, S.G. (1990). Adapted solution of a backward stochastic differential equation. Systems \& Control Letters, Vol. 14, 55-61.

[14] Pham, H. (1998). Optimal stopping of controlled jump diffusion processes: A viscosity solution approach. Journal of Mathematical Systems, Estimations and Control, Vol. 8, No. 1, $1-27$.

[15] Situ, R. (2005). Theory of Stochastic Differential Equations with Jumps and Applications. Springer, New York.

[16] Tang, S.J. \& Li, X.J. (1994). Necessary conditions for optimal control of stochastic systems with random jumps. SIAM Journal on Control and Optimization, Vol. 32, No. 5, 1447-1475.

[17] Yong, J.M. (1992). Dynamic Programming Method and the Hamilton-Jacobi-Bellman Equations (in Chinese). Shanghai Science and Technology Press, Shanghai.

[18] Yong, J.M. \& Zhou, X.Y. (1999). Stochastic Controls: Hamiltonian Systems and HJB Equations. Springer-Verlag, New York.

[19] Zhou, X.Y. (1990). The connection between the maximum principle and dynamic programming in stochastic control. Stochastics $\mathcal{E}$ Stochastics Reports, Vol. 31, 1-13. 